\title{
Is fintech good for bank performance? The case of mobile money in the East African Community*
}

\author{
Serge Ky ${ }^{a}$, b, , Clovis Rugemintwari ${ }^{\text {b* }}$, and Alain Sauviat ${ }^{b}$ \\ ${ }^{a}$ Université de Ouahigouya, Laboratoire d'Analyse et de Politique Économiques, Burkina Faso \\ ${ }^{b}$ Université de Limoges, Laboratoire d'Analyse et de Prospective Économiques, France
}

\section{This version: 5 June 2019.}

\begin{abstract}
Mobile money, a technology-driven innovation in financial services, has profoundly penetrated the financial landscape in Sub-Saharan Africa, including banks. Yet, besides anecdotal evidence, little is known about whether mobile money adoption enhances or worsens bank performance. Combining hand-collected data with balance sheet data from Bankscope for a panel of 170 financial institutions over the period 2009-2015, we find a strong positive and significant relationship between the time elapsed since banks' adoption of mobile money and their performance considering an array of proxies of bank profitability, efficiency and stability. In further investigations, we show how bank specialization and size alter such an association. Our results are robust to using instrumental variables, controlling for bank and macro level confounding factors, bank fixed effects and considering alternative measures of bank performance and mobile money adoption. Furthermore, we show that enhanced income diversification and broadened access to deposits are possible channels through which banks involved in mobile money improve their performance. Overall, our findings highlight the bright side of cooperation between banks and mobile network operators in the provision of mobile money. (JEL Classification G02, G21, G23)
\end{abstract}

Keywords: Fintech, Mobile money, Innovation, Bank performance, East African Community

\footnotetext{
*An earlier version of the paper was presented at the LAPE Bank Seminars. We are grateful to Ruth Tacneng, Céline Meslier, Isabelle Distinguin, Laetitia Lepetit and other participants for their constructive comments and suggestions. The usual disclaimer applies.

* Corresponding author. Tel: + 335551492 08. E-mail address: clovis.rugemintwari@unilim.fr
} 


\section{Introduction}

In most developing countries, formal financial institutions have been failing large swaths of society and nowhere is this truer than in Sub-Saharan Africa where only about a third has access to formal financial services according to the latest estimates from the World Bank (World Bank Group, 2018). At the same time, however, technology-driven innovation in financial serviceshenceforth fintech ${ }^{1}-$ is increasingly reshaping the African financial and banking landscape as never before (Sy et al., 2019). There is a large consensus that fintech is benefiting financially excluded people and underserved consumers around the globe and that this trend may continue over the next decade in all key financial respects such as savings, credits and investments (Jagtiani and John, 2018; IMF, 2018).

Although "Africa" and "innovation" are rarely associated, the leading position worldwide of the African continent in mobile money is starting to challenge this long-held perception. SubSaharan Africa played a pivotal role in mobile money emergence and development as shown in Van Der Boor et al. (2014). The authors challenge the widely-held view that the "North" is the unique source of new technology. They offer the first quantitative empirical study of the sources of innovation in mobile financial services and show that user-innovators in this field come from less-developed countries with a long-standing unfilled need of inexpensive banking services for the poor. Over the last decade, mobile money has grown rapidly following the tremendous penetration of mobile phones; for instance, the number of users has risen from $38 \%$ in 2009 to $72 \%$ in 2017 in Sub-Saharan Africa (The World Bank, 2019). This unprecedented usage of mobile phones creates an opportunity for the massive population who own a mobile phone but no bank account to be connected to the financial system ${ }^{2}$. Consistent with the very notion that at the core of fintech is the use of technology to provide new and improved financial services (Thakor, 2019), mobile money technology allows users to access financial services such as money transfers, payments, savings, insurance and digital credit. These financial operations are conducted through

\footnotetext{
${ }^{1}$ It is worth noting that throughout the paper, the terminology fintech is used alternatively for financial technology (mobile money in our case) or its provider when we refer to bank-fintech cooperation. The Financial Stability Board adopted a rather broad definition due to the rapidity and fluidity of fintech developments by pointing to "technologically-enabled financial innovation that could result in new business models, applications, processes, or products with an associated material effect on financial markets and institutions and the provision of financial services" (FSB, 2017, p.7).

${ }^{2}$ Cook and McKay (2017) contend that a large portion of mobile money users in Kenya is made up of those who had been unbanked. Furthermore, following the launch of M-Pesa, Safaricom's mobile money service, financial inclusion increased from 27 percent in 2006 to over 75 percent in 2016.
} 
mobile networks where cash-in cash-out services are provided by small business outlets better known as agents. The mobile money account can therefore be accessed without having an account at a financial institution but mobile money users who already possess a bank account have the possibility to connect both accounts.

While there are challenges for financial institutions to compete with fintech firms, there are also opportunities for collaborations. Indeed, banks are increasingly forging partnerships with Mobile Network Operators (MNO hereafter) and taking part into this disruptive innovation of mobile money. Advancements in new technologies allow financial institutions to serve businesses and consumers without costly brick and mortar investments. A recent but growing literature is starting to look at cooperation between banks and fintech firms and its potential effect on credit access (Drasch et al., 2018; Jagtiani and Lemieux, 2018). However, it is worth noting that measuring innovation in the financial sector is more challenging because of the lack of data which hampers assessing its impacts (Beck et al., 2016). Moreover, to the best of our knowledge, the existent works explore the case of developed countries and are most of the time U.S.-centric, owing to the fact that financial innovations often originate in the U.S., and that studies of such innovations usually rely on U.S. data (Frame et al., 2018; Frame and White, 2004).

Our paper aims to bridge this gap. It investigates how banks' involvement in mobile money innovation affects their performance in the five partner states (Burundi, Kenya, Rwanda, Tanzania and Uganda) of the East African Community (EAC). Two main reasons guide us to choose this region as our case study. First, although Sub-Saharan Africa taken as a whole continues to position itself as the global epicentre of mobile money, there is a wide degree of cross-country difference and the EAC is clearly at the forefront of mobile money innovation, adoption and usage as shown in Figure A1 in the Appendix (Minischetti and Scharwatt, 2016). The speed and scale of mobile money adoption in the $\mathrm{EAC}^{3}$ has been unprecedented and its impacts considerable (Jack and Suri, 2016, 2014; Mas and Ng'weno, 2010a; Mbiti and Weil, 2016). Hence, the dominant position of the banking sector in the financial system and the widespread adoption of mobile money motivate us to focus on the EAC in assessing the impact of mobile money adoption on bank performance.

\footnotetext{
${ }^{3}$ The most well-known and successful example of mobile money innovation is M-Pesa launched in 2007 in Kenya. Lashitew et al. (2019) provide an excellent in-depth background on its emergence, development and diffusion.
} 
Second, cross-border interoperability in mobile money is widespread in the region probably driven by the deep integration ${ }^{4}$ that facilitates remittances, trade and social exchange (BFA, 2017).

This study extends the existent literature on the risks and opportunities associated with bank-fintech cooperation and makes several contributions. First, most empirical studies tackling mobile money adoption focus mainly on its impact on various socio-economic aspects (e.g.: Bharadwaj et al., 2019; Jack and Suri, 2014; Munyegera and Matsumoto, 2016). To our knowledge, this paper is the first to conduct analysis of banks' mobile money adoption by examining how forging partnerships with MNOs affects bank performance. Specifically, we test whether the implication of banks in the mobile money scheme affects their profitability, efficiency and stability using a wide range of proxies traditionally considered in the banking literature. Second, our paper also adds to the literature investigating the impacts of financial technology on bank performance. Following Fuentelsaz et al. (2009) and Scott et al. (2017), we compare the performance of adopters and non-adopters. In addition, we take into account the time elapsed from the moment of adoption. Furthermore, Scott et al. (2017) point out the lack of evidence on the effects of innovation on smaller financial institutions. Hence, we test whether bank size and specialization alter the relationship between bank involvement in mobile money and bank performance. Finally, we complement the literature that explores the determinants of bank profitability, efficiency and stability in the East African Community.

The rest of the paper is organized as follows. Section 2 presents the related literature and our research questions, and in section 3 we provide the methodology, including our model specification, variables and data. We report and discuss the results in section 4, assess their robustness in section 5 and discuss the potential mechanisms in section 6. In section 7 we conclude.

\section{Related literature and research questions}

Bank-fintech cooperation may entail several benefits. For banks, such a partnership may yield sizable advantages in terms of development of new customer segments, products, and services. It may also result in expanding into new markets, developing new capabilities, and accessing new technologies that generate new revenue streams and improve efficiencies. This is

\footnotetext{
${ }^{4}$ In fact, EAC is the most integrated region in Africa according to the Africa Regional Integration Index (Koami et al., 2016). It has already in place a customs union, a common market and aims at building a political federation with a single currency.
} 
important because banks are often associated with a lack of innovation either because of their stable market position or because they are subject to complex and heavy government regulations (Anagnostopoulos, 2018). In return, fintechs may mainly benefit from bank reputation but also from new sources of finance and infrastructures (Drasch et al., 2018; The Economist Unit Intelligence, 2015). Digital innovations, such as mobile money, that promote financial inclusion gives a new impetus to the banking sector to improve its relationship with customers and increase performance. As such, digitization provides opportunities for banks to enhance their customerinteractions, improve their decision-making, and implement new business models in a more costeffective and innovative way (BCBS, 2018; Hirt and Willmott, 2014). In addition, fintechs are thought to be quicker and more agile than traditional banks. Hence, they are supposed to enable banks to engage in further cost-cutting strategies since bank services remain expensive ${ }^{5}$ (Drasch et al., 2018; Philippon, 2018). While these benefits are general in nature and apply across the globe, they are expected to be greater in the less developed part of the world where access to formal finance is still a challenge (Jagtiani and John, 2018).

As regards to the case of EAC, although mobile network operators are the most active actors, banks play a crucial role in mobile money provision. Specifically, to launch mobile money services, mobile network operators have to build partnership with banks or other financial institutions with a banking license (Aron, 2017; UNCTAD, 2012). In this case, banks play the role of custodians for mobile money users by holding a "trust" account or "escrow" account deposits that match the full extent of electronic money in the name of mobile network operators (Aron, 2017; Greenacre and Buckley, 2014). Banks can use these additional funds to increase their lending and this is not different from the way banks use ordinary deposits. Thus, bank involvement in the mobile money scheme includes simply holding a trust/escrow account (passive), building partnership to launch mobile money services (active), or both. To exploit other potential benefits associated with mobile money, some banks for instance build partnership with MNOs to increase the number of bank ATM users for cash out functions. These interest and fee-generating activities constitute new sources of income that may potentially enhance bank profitability. In addition, several MNOs have recognisable brands that have been developed through extensive marketing and service provision; therefore, banks can leverage mobile money platforms to reach more people

\footnotetext{
${ }^{5}$ Philippon (2018) estimates that the unit cost of banking intermediation in the US remained at about $2 \%$ over more than a century. Therefore, enhancing cost-efficiency may partly explain the emergence of fintechs.
} 
in traditionally underserved areas at much lower cost. Furthermore, MNOs in EAC developed extensive agent networks to sell airtime and other products while bank presence is often limited to urban or highly populated areas (UNCTAD, 2012). MNOs' extensive network may hence enhance bank efficiency.

In terms of bank stability, the literature purports that financial technology can potentially strengthen financial stability by fostering financial inclusion, increasing diversification and transparency ${ }^{6}$ as well as allowing better risk assessment (Sy et al., 2019). According to Ahamed and Mallick (2019), financial inclusion improves finacial stability by accessing cheap retail deposits from a large clientele base, reducing financing constraints of SMEs and mitigating the post-lending moral hazard. They also find that higher bank branch outreach and a financial system with higher access to bank accounts per capita are likely to increase bank stability. Nevertheless, allowing non-financial institutions (MNOs) to provide financial services may increase risk shifting from telecom sector to the banking system ${ }^{7}$. In this context, we assume that the effects of bank involvement in mobile money scheme on their performance may depend on the net outcome of these potential benefits and drawbacks.

In addition to comparing adopters to non-adopters to investigate the effect of mobile money adoption on bank profitability, efficiency or stability, we also take into account the time elapsed since adoption as emphasized by (Fuentelsaz et al., 2009). In fact, the initial adoption of mobile money may only indicate the point in time after which a bank is experimenting with it, but not the current moment in which mobile money is growing and its impacts on bank performance start to materialise. As such, research on financial technology adoption shows that an innovation evolves dynamically within the firm, following a diffusion process that starts with the adoption of the technology and requires time to be completed (Fuentelsaz et al., 2003). Thus, some delay in the achievement of the predicted performance gains should be expected because benefits stemming from a new technology are rarely fully achieved at the moment of adoption. Therefore, we hypothesize that longer ${ }^{8}$ bank-MNO partnership translates to better bank performance.

\footnotetext{
${ }^{6}$ Less face-to-face contact with bank officials reduces corruption and promotes efficient resource allocation.

${ }^{7}$ Kirilenko and Lo (2013) sums up such potential vulnerabilities as follows: "whatever can go wrong will go wrong faster and bigger when computers are involved".

${ }^{8}$ Note that mobile money is a relatively recent innovation of about a decade. Our sample period only covers 7 years; it runs from 2009 (such that for each country we have at least a financial institution in partnership with an MNO) to 2015.
} 
The literature also highlights other factors that equally matter when investigating the potential impact of financial technology adoption on firm performance, such as features of the technology, competition and differences in the characteristics of involved firms (Karshenas and Stoneman, 1993). For the purpose of our study and based on the structure and availability of our data, we take into consideration bank specialization and bank size (Scott et al., 2017). The failure of M-Kesho (launched in March 2010) vs. the spectacular success of its successor M-Shwari (launched in November 2012) is one of many anecdotal evidences that illustrate how decisive the sizes of the bank and MNOs involved in a partnership might be. In fact, M-Kesho and M-Shwari are digital credit products similar in every aspect except that Safaricom partnered with the largest bank in Kenya (Equity bank) to launch the former while for the later, the partner, CBA bank, was a small largely-unknown bank to the average Kenyan prior the partnership. A frequently cited reason behind the failure of M-Kesho mobile money service is that Equity bank and Safaricom perceived each other as main competitors and failed to define the partnership in a way that satisfied both companies. This phenomenon where a partnership between similarly sized organizations proved difficult, while a partnership between small and large entities succeeded is not rare and has propelled some to ask whether cooperation between equals in this area was even desirable (Cook and McKay, 2017; Flaming et al., 2013).

To sum it up, the foregoing discussion lead to the following research questions that our paper aims to address:

i. Does Bank-MNO partnership in mobile money provision affect bank profitability, efficiency and stability? We distinguish partnership as a point in time from the number of years of partnership since inception.

ii. Do these effects, if any, depend on the size and/or the specialization of the banks?

\section{Data, methodology and variables}

\subsection{Data sources}

We collect data on a sample of financial institutions in the EAC for the period 2009 to 2015 from a number of sources. The data on financial institutions' balance sheets, income statement are from the Bureau Van Djik (BvD) Bankscope database. We first collect the data of all financial institutions available in the database irrespective of whether they are listed or not and then we 
eliminate financial institutions with consolidated financial statement unless the unconsolidated data have no information. We also remove financial institutions with missing data for all the variables. Therefore, we obtain an unbalanced panel dataset comprising a final sample of 170 financial institutions operating in the five countries of the EAC, among which 153 are bank institutions split as follows: 8 are in Burundi, 66 in Kenya, 9 in Rwanda, 39 in Tanzania and 31 in Uganda. Data regarding financial institutions' involvement in mobile money are not readily available. We hence hand collected them by screening their websites and exploiting the GSMA tracker to identify financial institutions included in our sample that are involved in mobile money scheme. Afterwards, we track for each the number of years of its partnership with an MNO in the provision of mobile money (see Table A.6B in Appendix). Additional data on macroeconomic factors that may influence the financial institutions' performance are collected from the Global Financial development and World Development Indicators database of the World Bank. Finally, we collect data on the number of registered mobile money users and the value of mobile money transactions from the Financial Access Survey database of the International Monetary Fund.

\subsection{Model specification}

To address our research questions, we conduct estimations using the panel data fixed effects regression approach with two types of specifications. The first specification (equation 1) estimates the effects of mobile money on bank performance, while the second specification (equation 2) includes interaction terms to take into account bank size (small versus large).

$$
\begin{gathered}
Y_{i t}=\alpha_{1} M M_{i t}+\alpha_{2} X_{i t}+\eta_{i}+T_{t}+\varepsilon_{i t} \\
Y_{i t}=\beta_{1} M M_{i t}+\beta_{2} S M A L L_{i t}+\beta_{3} M M_{i t} \times S M A L L_{i t}+\beta_{4} X_{i t}+\eta_{i}+T_{t}+\omega_{i t}
\end{gathered}
$$

In our equations (1 and 2), $Y_{i t}$ represents the performance of bank $i$ at year $t . M M_{i t}$ is our independent variable of interest and stands alternatively for the status of bank $i$ 's implication in the mobile money system at time $t$ and the time elapsed since its mobile money adoption. $X_{i c t}$ denotes the vector of control variables including bank-specific and macroeconomic variables. $\eta_{i}$ is our set of bank fixed effects, and we include time dummies, $T_{t}$, to control for macroeconomic shocks. In equation (2), SMALL $L_{i t}$ is a dummy variable indicating bank size. It is equal to one if the bank is 
small and zero, otherwise. The coefficient $\boldsymbol{\beta}_{1}$ measures the effect of mobile money adoption for large banks while $\left(\boldsymbol{\beta}_{2}+\boldsymbol{\beta}_{3}\right)$ represents the total effect of mobile money for small banks.

In the robustness section, we tackle the concern that our main variable of interest, $M M$, may possibly be endogenous. The issue of simultaneity bias may arise from the fact that well performing banks may be more likely to build partnerships with mobile network operators to get involved in mobile money innovation. However, banks that perform poorly may equally view partnerships with an MNO as a way to improve their performance. This potential endogeneity issue cannot be completely dealt with in this case because devising good instruments is a daunting task as emphasized by Scott et al. (2017). Nevertheless, we check the robustness of our results. We follow previous literature (Demirgüç-Kunt and Huizinga, 2010; Fuentelsaz et al., 2012, 2009; Liang et al., 2013) and re-estimate our equations (1) and (2) by using the lagged value of MM, $M M_{i, t-1}$. In addition, we also use two country-level indicators of mobile money adoption as alternatives.

\subsection{Dependent variables: measures of bank performance}

Bank profitability, efficiency and stability are the most commonly considered dimensions in the empirical banking literature to measure bank performance ${ }^{9}$ (Ahamed and Mallick, 2019; Beccalli, 2007; Berger et al., 2010; Meslier et al., 2014; Saghi-Zedek, 2016; Scott et al., 2017). To evaluate bank profitability, we use two main variables (supplemented with two alternative variables in the robustness checks, see section 5): the return on assets (ROA) and the return on equity (ROE). ROA captures how effectively a bank utilizes its assets to generate income and is defined as net income divided by total assets while ROE, which is equal to net income divided by total equity, measures how well the bank manages resources invested by its shareholders. As regards to bank efficiency, we use the ratio COST/INCOME. Two alternative proxies are considered in the robustness check, namely the ratios of Non-interest expenses to average asset (NIEAA) and Nonoperating items and taxes to average assets (NOITAA). Finally, bank stability is measured by the z-score that we calculate as follows:

$Z-S C O R E_{i t}=\frac{R O A_{i t}+E Q A_{i t}}{\sigma\left(R O A_{i t}\right)}$

\footnotetext{
${ }^{9}$ We focus on accounting-based measures of performance because they are easy to compute, suitable when analyzing banks operating in developing countries and preferred when evaluating the impact of information systems investments on the banking sector's financial performance (Bitar et al., 2018; Fuentelsaz et al., 2012).
} 
Where $E Q A_{i t}$ is equity to assets ratio, and $\sigma\left(R O A_{i t}\right)$ is the standard deviation of return on assets. This score can be interpreted as the number of standard deviations below the mean by which return would have to drop before all equity in the bank gets depleted. The higher the ratios are, the better is bank performance for all our proxies except those related to bank efficiency, where lower values indicate higher efficiency.

\subsection{Explanatory variables: bank involvement in mobile money}

The main variable of interest in this study is bank involvement in mobile money, which may be passive (i.e. simply holds MNO's deposits in a trust account), active (mobile money services' provision in partnership with an MNO), or both. As indicated earlier, the involvement is assessed both as the bank's status of involvement at a point in time and as the number of years of Bank-MNO partnership since inception. For the former, we construct a dummy variable, which is equal to one if the bank has an existing partnership with a mobile network operator at time $t$, and zero, otherwise. For the latter, the time elapsed since a bank-MNO partnership came into existence is a categorical variable that takes the values from zero to nine whereby zero indicates that the bank has never been involved in mobile money and nine corresponds to nine years of bank involvement in mobile money. This measure allows us to capture the potential benefits that banks may accumulate over time through their partnership with the mobile network operator in launching mobile money and/or providing mobile money financial services. 
Table 1. Definitions of variables ${ }^{10}$.

\begin{tabular}{|c|c|c|c|c|c|c|c|}
\hline Variable & & Obs. & Mean & $\begin{array}{l}\text { Std. } \\
\text { Dev. }\end{array}$ & Min & Max & Source \\
\hline \multicolumn{8}{|l|}{ Dependent variable } \\
\hline ROA & Return on assets $=($ Net Income $/$ Total Assets $) * 100$ & 650 & 1.25 & 4.37 & -32.54 & 26.63 & Bankscope \\
\hline ROE & Return on equity $=($ Net Income $/$ Equity $) * 100$ & 650 & 7.15 & 31.81 & -567.80 & 76.55 & Bankscope \\
\hline Z-SCORE & Sum of average return on assets and equity ratio divided by standard deviation of return on assets & 648 & 29.87 & 40.14 & -1.45 & 386.60 & Bankscope \\
\hline COST/INCOME & Cost to income ratio, (Operating cost/total assets) $* 100$ & 623 & 76.97 & 66.17 & 5.75 & 876.84 & Bankscope \\
\hline RAROA & Return on assets divided by its three-year rolling- window standard deviation. & 650 & 0.28 & 0.95 & -7.94 & 6.10 & Bankscope \\
\hline NIM & Net interest margin ratio, (Net interest revenue/total assets) $* 100$ & 626 & 6.19 & 3.78 & -0.44 & 41.38 & Bankscope \\
\hline NIEAA & Non-interest expenses to average assets ratio. Higher value indicates higher cost. & 629 & 8.92 & 6.92 & 0.22 & 68.61 & Bankscope \\
\hline NOITAA & Non-operating items and taxes to average assets ratio. Higher value indicates higher cost. & 609 & -0.65 & 1.46 & $'-8.58$ & 13.85 & Bankscope \\
\hline Z-SCORE (3y) & $\begin{array}{l}\text { Sum of average return on assets and equity ratio divided by the three-year rolling- window standard deviation of } \\
\text { return on assets. }\end{array}$ & 650 & 0.32 & 0.96 & -7.86 & 6.32 & Bankscope \\
\hline Z-SCORE (5y) & $\begin{array}{l}\text { Sum of average return on assets and equity ratio divided by the five-year rolling- window standard deviation of } \\
\text { return on assets. }\end{array}$ & 650 & 0.32 & 0.94 & -7.09 & 6.61 & Bankscope \\
\hline \multicolumn{8}{|c|}{ Variable of interest (Mobile money - MM) } \\
\hline $\begin{array}{l}\text { Bank-MNO partnership ( } 1 \\
\text { if yes, } 0 \text { otherwise) }\end{array}$ & Banks involved in mobile money scheme takes the value 1 if there is a Bank-MNO partnership, and 0 otherwise. & 1071 & 0.11 & 0.32 & 0 & 1 & $\begin{array}{l}\text { Hand- } \\
\text { collected }\end{array}$ \\
\hline $\begin{array}{l}\text { Number of years of Bank- } \\
\text { MNO partnership since } \\
\text { inception }\end{array}$ & $\begin{array}{l}\text { Number of years elapsed since Bank-MNO partnership started providing mobile money services. It is a categorical } \\
\text { variable that takes the values from } 0 \text { to } 9 \text {, whereby } 0 \text { indicates that the bank has never been involved in Bank- } \\
\text { MNO partnership and } 9 \text { corresponds to } 9 \text { years of bank involvement in Bank-MNO partnership. }\end{array}$ & 1,071 & 0.73 & 1.80 & 0 & 9 & $\begin{array}{l}\text { Hand- } \\
\text { collected }\end{array}$ \\
\hline MMU & $\begin{array}{l}\text { logarithm (Number of accounts with a resident Mobile Money System Payment that is primarily accessed by a } \\
\text { mobile phone and is useable or has been used for mobile money transactions) }\end{array}$ & 1,006 & 16.41 & 1.11 & 12.21 & 17.80 & FAS \\
\hline MMT & $\begin{array}{l}\text { logarithm (Total amount of all mobile money transactions carried out). Calculated as = (value of mobile money } \\
\text { transactions) } * 100 / \text { GDP }\end{array}$ & 997 & 14.96 & 2.67 & 6.14 & 17.13 & FAS \\
\hline \multicolumn{8}{|c|}{ 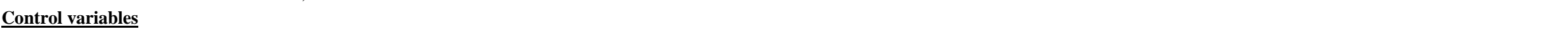 } \\
\hline SIZE & Bank size $=\ln ($ Total Assets $)$ & 652 & 12.26 & 1.29 & 8.13 & 15.34 & Bankscope \\
\hline LLP & Ratio of Loan loss provision to total asset & 591 & 0.93 & 1.68 & -3.11 & 17.71 & Bankscope \\
\hline TL_TA & Ratio of total loans to total assets & 638 & 51.71 & 14.11 & 0.89 & 98.14 & Bankscope \\
\hline ASSET Growth & Assets growth & 528 & 17.39 & 32.10 & -85.34 & 477.55 & Bankscope \\
\hline NII & Non-interest income $=$ Other Operating Income $/$ Operating Income & 647 & 0.38 & 0.20 & -0.48 & 1.11 & Bankscope \\
\hline OBS & Off-balance sheet $=$ Off Balance Sheet Items $/$ Total Assets & 590 & 0.16 & 0.15 & 0.00 & 1.45 & Bankscope \\
\hline GDP Growth & Gross Domestic Product growth & 1071 & 5.83 & 1.75 & -3.90 & 9.44 & WDIs \\
\hline INFL & Inflation growth rate & 1071 & 8.01 & 4.53 & -5.32 & 21.52 & WDIs \\
\hline SMALL & $\begin{array}{l}\text { a dummy variable that takes the value } 1 \text { if the bank total assets are less than the median (Small bank), and } 0 \\
\text { otherwise (Large bank) }\end{array}$ & 1,071 & 0.31 & 0.46 & 0 & 1 & Bankscope \\
\hline
\end{tabular}

Note: FAS stands for Financial Access Survey (International Monetary Fund), WDIs stands for World Development Indicators (World Bank Group).

${ }^{10}$ The correlation matrix is reported in Table A.7 in the Appendix. 


\subsection{Control variables: bank and country level variables}

Based on the existing literature (Berger et al., 2010; Köster and Pelster, 2017; Meslier et al., 2014; Mostak Ahamed, 2017; Shaban and James, 2014), we use a number of banks' characteristics and macroeconomic factors that may affect bank performance. We use the logarithm of total assets (SIZE) to control for size-induced performance differences in banks. We use the ratio of loan loss provision to total asset (LLP) to control for loan quality of individual bank. The LLP ratio can affect banks' performance as banks can use loan provisions to smooth their earnings. We control for a bank's asset composition by using the ratio of total loans to total assets (TL_TA). We include annual growth rate of total assets (ASSET Growth) to take into account how business growth can affect bank performance while the effect of diversification is controlled by using the share of non-interest income to total operation income (NII). Finally, we take into account the effect on bank performance that may stem from the off-balance sheet activities (OBS). Macroeconomic characteristics are controlled for by using gross domestic product growth (GDP Growth) and inflation rates (INFL).

\section{Empirical results}

Table 2, Panel A, reports our regression results of the effect of mobile money adoption on bank performance using our first specification (equation (1)) and considering the whole sample of banks. The results, which indicate a solid positive link between banks' involvement in the mobile money scheme and their performance, provide the first empirical evidence of the bright side of mobile money adoption at the bank level. However, our findings also reveal that what really matters is not the involvement per se but rather the time elapsed since the partnership came into existence. Precisely, we find that the coefficients associated with the variable involvement of banks in mobile money, $M M$, are not significant (columns (1) - (3)) or significant at only 10\% (column (4) when proxied by the dummy variable whereas they are strongly significant in the case of the categorical variable (columns (5) - (8)). These findings are consistent with those of Fuentelsaz et al. (2003) which show that there is a delay before the achievement of the expected performance gains stemming from the adoption of automated teller machines (ATM) in the case of Spanish savings banks. Similarly, they are in line with those of Scott et al. (2017) who emphasize that the effects of SWIFT adoption on bank profitability are much stronger in the long-run. Our results also echo empirical findings in the banking literature highlighting the positive effects of financial inclusion 
and/or bank diversification on performance (Ahamed and Mallick, 2019; Han and Melecky, 2014). In our case, the involvement in mobile money scheme may enable banks to cater to new segments of customers and thus diversify further their income streams by engaging in activities less correlated with their core ones. In a similar vein, banks' partnership with mobile network operators may plausibly result in new cost-effective and innovative ways such as allowing customers to perform their banking transactions without visiting banks' agencies. 
Table 2. Mobile money and bank performance in the East African Community.

\begin{tabular}{|c|c|c|c|c|c|c|c|c|}
\hline \multirow{3}{*}{ Panel A } & \multicolumn{8}{|c|}{ ALL BANKS } \\
\hline & \multicolumn{4}{|c|}{ Bank-MNO partnership (1 if yes, 0 otherwise) } & \multicolumn{4}{|c|}{ Number of years of Bank-MNO partnership since inception } \\
\hline & $\begin{array}{l}\text { ROA } \\
\text { (1) }\end{array}$ & $\begin{array}{l}\text { ROE } \\
(2)\end{array}$ & $\begin{array}{l}\text { COST/INCOME } \\
\text { (3) }\end{array}$ & $\begin{array}{l}\text { Z-SCORE } \\
\text { (4) }\end{array}$ & $\begin{array}{l}\text { ROA } \\
(5)\end{array}$ & $\begin{array}{l}\text { ROE } \\
(6)\end{array}$ & $\begin{array}{l}\text { COST/INCOME } \\
\text { (7) }\end{array}$ & $\begin{array}{l}\text { Z-SCORE } \\
\text { (8) }\end{array}$ \\
\hline \multirow[t]{2}{*}{ MM } & 0.650 & 4.466 & -9.500 & $0.429 *$ & $0.333 * * *$ & $1.862 * *$ & $-6.367 * * *$ & $0.982 * * *$ \\
\hline & $(0.454)$ & $(3.541)$ & $(6.934)$ & $(0.249)$ & $(0.105)$ & $(0.826)$ & $(1.594)$ & $(0.332)$ \\
\hline \multirow[t]{2}{*}{ SIZE } & $3.711 * * *$ & $25.429 * * *$ & $-75.261 * * *$ & $0.519^{*}$ & $3.968 * * *$ & $26.999 * * *$ & $-79.684 * * *$ & -2.063 \\
\hline & $(0.485)$ & $(3.784)$ & $(7.410)$ & $(0.266)$ & $(0.476)$ & $(3.739)$ & $(7.215)$ & (1.501) \\
\hline \multirow[t]{2}{*}{$\mathbf{L L P}$} & $-0.444 * * *$ & $-2.919 * * *$ & -0.190 & $-0.240 * * *$ & $-0.443 * * *$ & $-2.908 * * *$ & -0.179 & $-0.723^{* * *} *$ \\
\hline & $(0.061)$ & $(0.474)$ & $(0.928)$ & $(0.033)$ & $(0.060)$ & $(0.471)$ & $(0.908)$ & $(0.189)$ \\
\hline \multirow[t]{2}{*}{ TL_TA } & -0.002 & 0.064 & -0.014 & 0.004 & -0.003 & 0.062 & -0.014 & $-0.084 *$ \\
\hline & $(0.015)$ & $(0.114)$ & $(0.223)$ & $(0.008)$ & $(0.014)$ & $(0.113)$ & $(0.219)$ & $(0.046)$ \\
\hline \multirow[t]{2}{*}{ ASSETS Growth } & -0.005 & 0.035 & $0.243^{* * *}$ & 0.000 & $-0.006^{*}$ & 0.031 & $0.253^{* * *} *$ & -0.016 \\
\hline & $(0.003)$ & $(0.025)$ & $(0.050)$ & $(0.002)$ & $(0.003)$ & $(0.025)$ & $(0.048)$ & $(0.010)$ \\
\hline \multirow[t]{2}{*}{ NII } & -1.449 & $-14.529 *$ & $40.425 * * *$ & 0.337 & -1.520 & $-14.682 *$ & $42.648^{* * * *}$ & 0.208 \\
\hline & $(0.991)$ & $(7.730)$ & (15.138) & $(0.544)$ & (0.974) & $(7.649)$ & $(14.761)$ & (3.072) \\
\hline \multirow[t]{2}{*}{ OBS } & -0.268 & 3.719 & -0.689 & 0.722 & -0.024 & 5.074 & -5.384 & 1.665 \\
\hline & $(0.861)$ & (6.719) & $(13.157)$ & $(0.473)$ & $(0.855)$ & $(6.714)$ & (12.957) & $(2.696)$ \\
\hline \multirow[t]{2}{*}{ GDP Growth } & $0.289 * * *$ & $2.164 * * *$ & $-3.323 * * *$ & $0.172 * * *$ & $0.284 * * *$ & $2.133^{* * *} *$ & $-3.234 * * *$ & $0.682 * * *$ \\
\hline & $(0.078)$ & $(0.606)$ & (1.186) & $(0.043)$ & $(0.077)$ & $(0.603)$ & (1.163) & $(0.242)$ \\
\hline \multirow[t]{2}{*}{ INFL } & $0.074 * * *$ & $0.679 * * *$ & $-1.250 * * *$ & $0.030 * *$ & $0.078 * * *$ & $0.700^{* * *} *$ & $-1.310 * * *$ & 0.115 \\
\hline & $(0.024)$ & $(0.191)$ & $(0.374)$ & $(0.013)$ & $(0.024)$ & $(0.190)$ & $(0.367)$ & $(0.076)$ \\
\hline \multirow[t]{2}{*}{ Constant } & $-45.249 * * *$ & $-317.777 * * *$ & $1,003.214^{* * * *}$ & -4.908 & $-48.368 * * *$ & $-336.712 * * *$ & $1,057.513 * * *$ & $51.809^{* * * *}$ \\
\hline & $(6.101)$ & $(47.605)$ & $(93.228)$ & $(3.352)$ & $(6.009)$ & $(47.183)$ & $(91.051)$ & $(18.946)$ \\
\hline Observations & 466 & 466 & 466 & 466 & 466 & 466 & 466 & 466 \\
\hline R2 within & 0.307 & 0.258 & 0.369 & 0.206 & 0.323 & 0.265 & 0.393 & 0.100 \\
\hline R2 between & 0.253 & 0.307 & 0.258 & 0.124 & 0.253 & 0.307 & 0.251 & 0.0316 \\
\hline \multirow{4}{*}{$\begin{array}{l}\text { Number of } \\
\text { banks } \\
\text { Fixed effects } \\
\text { Time dummies }\end{array}$} & 103 & 103 & 103 & 103 & 103 & 103 & 103 & 103 \\
\hline & YES & YES & YES & YES & YES & YES & YES & YES \\
\hline & YES & YES & YES & YES & YES & YES & YES & YES \\
\hline & COMMER( & IAL BANKS & & & & & & \\
\hline \multirow[t]{2}{*}{ Panel B } & Bank-MNO & artnership (1 & yes, 0 otherwise) & & Number of & ars of Bank-N & JO partnership sinc & ception \\
\hline & $\begin{array}{l}\text { ROA } \\
\text { (1) }\end{array}$ & $\begin{array}{l}\text { ROE } \\
(2)\end{array}$ & $\begin{array}{l}\text { COST/INCOME } \\
\text { (3) }\end{array}$ & $\begin{array}{l}\text { Z-SCORE } \\
\text { (4) }\end{array}$ & $\begin{array}{l}\text { ROA } \\
(5)\end{array}$ & $\begin{array}{l}\text { ROE } \\
(6)\end{array}$ & $\begin{array}{l}\text { COST/INCOME } \\
\text { (7) }\end{array}$ & $\begin{array}{l}\text { Z-SCORE } \\
\text { (8) }\end{array}$ \\
\hline MM & 0.309 & 1.039 & -6.454 & 0.281 & $0.262 * *$ & 1.417 & $-6.359 * * *$ & $1.271 * * *$ \\
\hline & $(0.502)$ & $(3.826)$ & $(7.930)$ & $(0.272)$ & $(0.115)$ & $(0.881)$ & $(1.798)$ & $(0.373)$ \\
\hline SIZE & $3.846^{* * *}$ & $27.644 * * *$ & $-76.452 * * *$ & $0.583 * *$ & $4.031 * * *$ & $28.509 * * *$ & $-80.716^{* * * *}$ & -1.716 \\
\hline & $(0.516)$ & $(3.936)$ & $(8.158)$ & $(0.280)$ & $(0.504)$ & $(3.855)$ & $(7.863)$ & $(1.632)$ \\
\hline $\mathbf{L L P}$ & $-0.760 * * *$ & $-4.824 * * *$ & -0.617 & $-0.394 * * *$ & $-0.750 * * *$ & $-4.773 * * *$ & -0.861 & $-0.833 * * *$ \\
\hline & $(0.096)$ & $(0.729)$ & $(1.510)$ & $(0.052)$ & $(0.095)$ & $(0.725)$ & $(1.479)$ & $(0.307)$ \\
\hline TL_TA & -0.005 & 0.013 & 0.034 & 0.004 & -0.006 & 0.009 & 0.056 & $-0.097 *$ \\
\hline & $(0.015)$ & $(0.117)$ & $(0.244)$ & $(0.008)$ & $(0.015)$ & $(0.117)$ & $(0.239)$ & $(0.050)$ \\
\hline ASSETS Growth & -0.005 & $0.045 *$ & $0.233 * * *$ & 0.000 & $-0.006 *$ & $0.045^{*}$ & $0.238^{*} * *$ & -0.016 \\
\hline & $(0.003)$ & $(0.026)$ & $(0.055)$ & $(0.002)$ & $(0.003)$ & $(0.026)$ & $(0.053)$ & $(0.011)$ \\
\hline NII & -0.225 & -4.642 & $43.939 * *$ & $1.151^{*}$ & -0.251 & -4.948 & $44.847 * * *$ & 2.239 \\
\hline & (1.101) & $(8.394)$ & $(17.398)$ & $(0.598)$ & $(1.085)$ & $(8.306)$ & $(16.941)$ & $(3.516)$ \\
\hline OBS & -0.517 & 9.571 & -3.193 & 0.841 & -0.281 & 10.808 & -8.864 & 1.466 \\
\hline & (1.096) & $(8.355)$ & $(17.318)$ & $(0.595)$ & $(1.090)$ & $(8.346)$ & $(17.024)$ & $(3.533)$ \\
\hline GDP Growth & $0.337 * * *$ & $2.563 * * *$ & $-3.794 * * *$ & $0.179 * * *$ & $0.332 * * *$ & $2.536^{* * *} *$ & $-3.680 * * *$ & $0.765^{* * *}$ \\
\hline & $(0.084)$ & $(0.642)$ & $(1.331)$ & $(0.046)$ & $(0.083)$ & $(0.639)$ & $(1.304)$ & $(0.271)$ \\
\hline INFL & $0.081 * * *$ & $0.721 * * *$ & $-1.231 * * *$ & $0.027^{*}$ & $0.085^{* * * *}$ & $0.738 * * *$ & $-1.309 * * *$ & 0.140 \\
\hline & $(0.027)$ & $(0.203)$ & $(0.420)$ & $(0.014)$ & $(0.026)$ & $(0.202)$ & $(0.412)$ & $(0.086)$ \\
\hline Constant & $-47.536^{* * *}$ & $-349.600 * * *$ & $1,019.903^{* * *} *$ & $-6.095^{*}$ & $-49.843^{* * *}$ & $-360.506 * * *$ & $1,073.252 * * *$ & $42.966^{* *}$ \\
\hline & $(6.540)$ & $(49.868)$ & $(103.363)$ & $(3.551)$ & $(6.404)$ & $(49.034)$ & $(100.016)$ & $(20.756)$ \\
\hline Observations & 381 & 381 & 381 & 381 & 381 & 381 & 381 & 381 \\
\hline R2 within & 0.363 & 0.316 & 0.390 & 0.248 & 0.374 & 0.322 & 0.415 & 0.116 \\
\hline R2 between & 0.298 & 0.357 & 0.272 & 0.148 & 0.292 & 0.352 & 0.260 & 0.0109 \\
\hline $\begin{array}{l}\text { Number of } \\
\text { banks }\end{array}$ & 84 & 84 & 84 & 84 & 84 & 84 & 84 & 84 \\
\hline Fixed effects & YES & YES & YES & YES & YES & YES & YES & YES \\
\hline Time dummies & YES & YES & YES & YES & YES & YES & YES & YES \\
\hline
\end{tabular}


As regards our control variables, the results are consistent with existing literature. The bank-level control variables SIZE and LLP indicate that an increase in bank size leads to an improvement in profitability, efficiency and stability while loan loss provision is negatively linked to bank performance. Turning to our country-level controls, both are highly significant and, as might be expected, a higher GDP growth rate is associated with a better bank performance. We also find that the inflation rate and bank performance are positively related.

To take bank specialization into consideration, we restrict our sample to commercial banks to check whether and how our earlier findings might be altered. This is important because the banking sector in EAC is dominated by commercial banks that play a critical role in the transmission mechanism of the monetary policy. Panel B of Table 2 discloses the results. Overall, the estimates show similar results but the positive association between bank involvement in mobile money scheme and bank profitability is less pronounced in this case. More precisely, we notice a drop in significance in column 5 when bank profitability is proxied by ROA and a loss of significance in column 6 when the ROE is considered. A plausible explanation for these results on commercial banks is provided by (Fuentelsaz et al., 2009). They argue that a new technology may have a stronger impact on firms' efficiency compared to profitability because as the technology is diffused to other firms, the adoption of the technology may simply lead to an increased competition resulting in negligible variations in profitability.

Finally, we check whether bank size (SMALL) affects the positive relationship uncovered between mobile money adoption and bank performance. Therefore, we split our sample into small and large banks based on the median of total assets of the banking sector per country. The computation of this median is constrained by the availability of data for each bank in each country. Two main conclusions emerge from the results reported in Panels A and B of Table 3. Considering large banks, we find that the results perfectly mimic those shown in Table 2: the longer the partnership is with an MNO, the better bank performance is. Regarding small banks, the results show that being involved in mobile money is strongly associated with both their profitability and efficiency irrespective of their degree of involvement in a partnership with an MNO. Precisely, the coefficients of both the dummy variable (columns (1)-(3) and the categorical variable (columns (5)-(7)) are significant at $1 \%$ level. These results are consistent with those of Dos Santos and Peffers (1995) and Scott et al. (2017) which show that the effect of technological innovation on profitability is higher on small firms than on larger firms because the former can adapt faster to it compared to 
larger ones that may be sluggish to respond due to their stable market position and legacy systems which require substantial changes. We do not find, however, any significant link between bank stability (Z-score) and mobile money adoption for small banks. These results may suggest that compared to large banks, the relative risks from financial technology adoption can be considerably higher for smaller banks due to their limited resources and lack of knowledge about technology management (Scott et al., 2017). 
Table 3. Mobile money and bank performance: Small vs. Large banks.

\begin{tabular}{|c|c|c|c|c|c|c|c|c|}
\hline \multirow{3}{*}{ Panel A } & \multicolumn{8}{|c|}{ ALL BANKS (Small vs Large Banks) } \\
\hline & \multicolumn{4}{|c|}{ Bank-MNO partnership (1 if yes, 0 otherwise) } & \multicolumn{4}{|c|}{ Number of years of Bank-MNO partnership since inception } \\
\hline & $\begin{array}{l}\text { ROA } \\
\text { (1) }\end{array}$ & $\begin{array}{l}\text { ROE } \\
\text { (2) }\end{array}$ & $\begin{array}{l}\text { COST/INCOME } \\
\text { (3) }\end{array}$ & $\begin{array}{l}\text { Z-SCORE } \\
(4)\end{array}$ & $\begin{array}{l}\text { ROA } \\
(5)\end{array}$ & $\begin{array}{l}\text { ROE } \\
(6)\end{array}$ & $\begin{array}{l}\text { COST/INCOME } \\
\text { (7) }\end{array}$ & $\begin{array}{l}\text { Z-SCORE } \\
(8)\end{array}$ \\
\hline MM & $\begin{array}{l}0.034 \\
(0.511)\end{array}$ & $\begin{array}{l}0.530 \\
(4.012)\end{array}$ & $\begin{array}{l}-1.769 \\
(7.872)\end{array}$ & $\begin{array}{l}0.332 \\
(0.286)\end{array}$ & $\begin{array}{l}0.278 * * * \\
(0.105)\end{array}$ & $\begin{array}{l}1.466^{*} \\
(0.826)\end{array}$ & $\begin{array}{l}-5.397 * * * \\
(1.572)\end{array}$ & $\begin{array}{l}1.019 * * * \\
(0.336)\end{array}$ \\
\hline SMALL & $\begin{array}{l}0.724 * * \\
(0.353)\end{array}$ & $\begin{array}{l}4.970^{*} \\
(2.767)\end{array}$ & $\begin{array}{l}-8.258 \\
(5.430)\end{array}$ & $\begin{array}{l}0.112 \\
(0.197)\end{array}$ & $\begin{array}{l}0.639^{*} \\
(0.350)\end{array}$ & $\begin{array}{l}4.306 \\
(2.757)\end{array}$ & $\begin{array}{l}-3.133 \\
(5.250)\end{array}$ & $\begin{array}{l}0.621 \\
(1.121)\end{array}$ \\
\hline MM x SMALL & $\begin{array}{l}1.533 * * \\
(0.646)\end{array}$ & $\begin{array}{l}9.773^{*} \\
(5.071)\end{array}$ & $\begin{array}{l}-19.285^{*} \\
(9.948)\end{array}$ & $\begin{array}{l}0.243 \\
(0.362)\end{array}$ & $\begin{array}{l}0.486 * * \\
(0.206)\end{array}$ & $\begin{array}{l}3.543 * * \\
(1.625)\end{array}$ & $\begin{array}{l}-12.485 * * * \\
(3.094)\end{array}$ & $\begin{array}{l}-0.848 \\
(0.660)\end{array}$ \\
\hline Total effect & $\begin{array}{l}2.257302 * * * \\
(0.630)\end{array}$ & $\begin{array}{l}14.743^{* * * *} \\
(4.946)\end{array}$ & $\begin{array}{l}-27.544 * * * \\
(9.705)\end{array}$ & $\begin{array}{l}0.355 \\
(0.353)\end{array}$ & $\begin{array}{l}1.125^{* * * *} \\
(0.348)\end{array}$ & $\begin{array}{l}7.849 * * * \\
(2.744)\end{array}$ & $\begin{array}{l}-15.618^{* * * *} \\
(5.224)\end{array}$ & $\begin{array}{l}0.227 \\
(1.115)\end{array}$ \\
\hline Controls included & YES & YES & YES & YES & YES & YES & YES & YES \\
\hline $\begin{array}{l}\text { Observations } \\
\text { R2 within } \\
\text { R2 between } \\
\text { Number of banks } \\
\text { Fixed effects } \\
\text { Time dummies } \\
\end{array}$ & $\begin{array}{l}466 \\
0.335 \\
0.240 \\
103 \\
\text { YES } \\
\text { YES } \\
\end{array}$ & $\begin{array}{l}466 \\
0.279 \\
0.289 \\
103 \\
\text { YES } \\
\text { YES } \\
\end{array}$ & $\begin{array}{l}466 \\
0.384 \\
0.253 \\
103 \\
\text { YES } \\
\text { YES } \\
\end{array}$ & $\begin{array}{l}466 \\
0.209 \\
0.117 \\
103 \\
\text { YES } \\
\text { YES } \\
\end{array}$ & $\begin{array}{l}466 \\
0.346 \\
0.244 \\
103 \\
\text { YES } \\
\text { YES } \\
\end{array}$ & $\begin{array}{l}466 \\
0.286 \\
0.295 \\
103 \\
\text { YES } \\
\text { YES } \\
\end{array}$ & $\begin{array}{l}466 \\
0.426 \\
0.244 \\
103 \\
\text { YES } \\
\text { YES } \\
\end{array}$ & $\begin{array}{l}466 \\
0.104 \\
0.027 \\
103 \\
\text { YES } \\
\text { YES } \\
\end{array}$ \\
\hline \multirow{3}{*}{ Panel B } & \multirow{2}{*}{\multicolumn{4}{|c|}{$\begin{array}{l}\text { COMMERCIAL BANKS (Small vs Large Banks) } \\
\text { Bank-MNO partnership (1 if ves, o otherwise) }\end{array}$}} & & & & \\
\hline & & & & & \multicolumn{4}{|c|}{ Number of years of Bank-MNO partnership since inception } \\
\hline & $\begin{array}{l}\text { ROA } \\
\text { (1) }\end{array}$ & $\begin{array}{l}\text { ROE } \\
(2)\end{array}$ & $\begin{array}{l}\text { COST/INCOME } \\
\text { (3) }\end{array}$ & $\begin{array}{l}\text { Z-SCORE } \\
(4)\end{array}$ & $\begin{array}{l}\text { ROA } \\
(5) \\
\end{array}$ & $\begin{array}{l}\text { ROE } \\
(6)\end{array}$ & $\begin{array}{l}\text { COST/INCOME } \\
(7)\end{array}$ & $\begin{array}{l}\text { Z-SCORE } \\
(8)\end{array}$ \\
\hline MM & $\begin{array}{l}-0.417 \\
(0.546)\end{array}$ & $\begin{array}{l}-3.351 \\
(4.196)\end{array}$ & $\begin{array}{l}2.384 \\
(8.685)\end{array}$ & $\begin{array}{l}0.263 \\
(0.304)\end{array}$ & $\begin{array}{l}0.211^{*} \\
(0.114)\end{array}$ & $\begin{array}{l}1.088 \\
(0.881)\end{array}$ & $\begin{array}{l}-5.489 * * * \\
(1.761)\end{array}$ & $\begin{array}{l}1.288 * * * \\
(0.378)\end{array}$ \\
\hline SMALL & $\begin{array}{l}0.751 * * \\
(0.378)\end{array}$ & $\begin{array}{l}4.932 * \\
(2.904)\end{array}$ & $\begin{array}{l}-11.954 * * \\
(6.011)\end{array}$ & $\begin{array}{l}0.013 \\
(0.210)\end{array}$ & $\begin{array}{l}0.692 * \\
(0.379)\end{array}$ & $\begin{array}{l}4.472 \\
(2.919)\end{array}$ & $\begin{array}{l}-6.572 \\
(5.838)\end{array}$ & $\begin{array}{l}0.331 \\
(1.252)\end{array}$ \\
\hline MM x SMALL & $\begin{array}{l}1.791 * * \\
(0.703)\end{array}$ & $\begin{array}{l}10.670 * * \\
(5.410)\end{array}$ & $\begin{array}{l}-20.714 * \\
(11.197)\end{array}$ & $\begin{array}{l}0.047 \\
(0.392)\end{array}$ & $\begin{array}{l}0.534 * * \\
(0.239)\end{array}$ & $\begin{array}{l}3.433^{*} \\
(1.842)\end{array}$ & $\begin{array}{l}-14.000 * * * \\
(3.683)\end{array}$ & $\begin{array}{l}-0.710 \\
(0.790)\end{array}$ \\
\hline Total effect & $\begin{array}{l}2.541 * * * \\
(0.702)\end{array}$ & $\begin{array}{l}15.602 * * * \\
(5.396)\end{array}$ & $\begin{array}{l}-32.668 * * * \\
(11.168)\end{array}$ & $\begin{array}{l}0.060 \\
(0.391)\end{array}$ & $\begin{array}{l}1.226 * * * \\
(0.386)\end{array}$ & $\begin{array}{l}7.905^{* * * *} \\
(2.972)\end{array}$ & $\begin{array}{l}-20.572 * * * \\
(5.944)\end{array}$ & $\begin{array}{l}-0.378 \\
(1.274)\end{array}$ \\
\hline Controls included & YES & YES & YES & YES & YES & YES & YES & YES \\
\hline Observations & 381 & 381 & 381 & 381 & 381 & 381 & 381 & 381 \\
\hline R2 within & 0.394 & 0.338 & 0.412 & 0.248 & 0.399 & 0.341 & 0.453 & 0.118 \\
\hline R2 between & 0.294 & 0.352 & 0.272 & 0.148 & 0.290 & 0.350 & 0.255 & 0.008 \\
\hline Number of banks & 84 & 84 & 84 & 84 & 84 & 84 & 84 & 84 \\
\hline Fixed effects & YES & YES & YES & YES & YES & YES & YES & YES \\
\hline Time dummies & YES & YES & YES & YES & YES & YES & YES & YES \\
\hline
\end{tabular}

Note: Dependent variables: Return on assets (ROA), Return on equity (ROE), Cost to income ratio (COST/INCOME), and the sum of return on assets and equity ratio divided by the standard deviation of return on assets (Z-SCORE). MM, involvement in mobile money, stands alternatively for Bank-MNO partnership that equals 1 if yes, 0 otherwise, and the number of years of Bank-MNO partnership since inception. SMALL is a dummy variable that takes the value 1 if the bank total assets are less than the median, and 0 otherwise. Robust standard errors are in brackets Controls included: SIZE is the logarithm of total asset; LLP is the ratio of loan loss provision over total assets; TL_TA is the ratio of total loans to total assets; ASSETS Growth is the growth rate of total assets; NII is the share of non-interest income over total operating income; OBS is the ratio of off-balance sheet over total assets; GDP Growth is the annual growth rate of gross domestic product; INFL is the annual growth rate of consumer price index. ***Significant at the $1 \%$ level, **Significant at the 5\% level, *Significant at the $10 \%$ level. 


\section{Robustness checks}

In this section, we consider several sensitivity tests to check the strength of our findings. First, we consider alternative measures of bank performance as dependent variables. For profitability, we consider return on assets divided by its three-year rolling-window standard deviation (RAROA) and net interest margin ratio (NIM). As regards to efficiency, we use noninterest expenses to average assets ratio (NIEAA) and non-operating items and taxes to average assets ratio (NOITAA). Finally, our measure of bank stability, z-score, is modified using threeyear and five-year rolling- window standard deviations (respectively Z-SCORE (3y) and Z-SCORE (5y)) (Beccalli, 2007; Bitar et al., 2018; Saghi-Zedek, 2016). Tables A.1A and A.1B report the results for our all our six alternative dependent variables. We find results consistent with our main findings indicating that the coefficients associated with bank involvement in mobile money proxied by the categorical variables are significant, except a slight drop in significance for our efficiency variables (Table A.1B). Our findings obtained taking into account bank size and bank specialization are confirmed as well. Specifically, consistent with our main findings, we notice that for small banks the length of involvement in mobile money does not matter: irrespective of the length, being involved in mobile money is associated with an improvement in bank performance. The only difference is that bank stability (proxied by Z-SCORE (3y) and Z-SCORE (5y)) are now positively and significantly affected. Second, we perform an instrumental variable analysis by following Demirgüç-Kunt and Huizinga (2010) and Liang et al. (2013). Thus, we re-estimate our equations ( 1 and 2 ) where the lagged bank involvement in mobile money $M M_{i, t-1}$, replaces the current year values $M M_{i, t}$. The results in Table A.2 show that all our earlier conclusions are unaltered. Moreover, we also consider country-level measures of mobile money adoption as alternatives to our main variable of interest suspected to be endogenous. In fact, effective benefit stemming from banks' involvement in mobile money provision requires individual access and usage of mobile money services. Hence, we use the number of mobile money accounts (MMU) and the value of mobile money transactions (MMT). The concern of potential reverse causality might no longer be the case as we consider the effect of country-level variables on bank-level measures of bank performance. The results show that both the number of accounts and the value of transactions of mobile money are, as might be expected, positively and negatively related to bank profitability and efficiency respectively (Table A.3A). These findings hold for large banks and commercial banks but not for small banks (Table A.3B). Third, we extend our analysis to all 
financial institutions operating in the EAC (Table A.4). Overall, we find results consistent with our previous findings. The only notable difference is that the coefficients associated with bank involvement measured as a dummy variable are now positive and significantly associated with bank profitability and stability. Given that microfinance institutions are comparatively smaller, this result amplifies our earlier findings on small banks showing that bank involvement in mobile money positively affects its performance irrespective of the time elapsed since mobile money adoption.

\section{Why banks' involvement in mobile money may improve their performance? Discussion of potential mechanisms}

In this section, we discuss potential mechanisms to figure out different channels through which banks' involvement in mobile money may improve their performance. Traditionally, banks provide cash-in/cash-out services via ATMs and bank branches. However, these solutions are too expensive to set up and operate in markets that have low-income or low-density populations. Therefore, we exploit the idea that bank-MNO partnership in mobile money provision may enable banks to leverage mobile money agent networks to reach those areas with limited population size or economic activity. Clearly, we investigate whether the improvement in bank performance found in our study is the result of enhanced income diversification and broadened access to financial services stemming from the cooperation between banks and mobile network operators in mobile money provision. Accordingly, the positive relationship shown between banks' involvement in mobile money and their performance is expected to be more pronounced for banks with low retail deposit funding or low income diversification. As a measure of deposit funding indicator, we use the short-term deposit funding. We compute two dummy variables, the first, HIGH deposit funding, indicates banks that have business model focusing more on deposit funding from customers with a deposit funding indicator that is higher than the sample mean. The second, LOW deposit funding share, reports banks that focus less on customer deposits funding and equals to one minus HIGH deposit funding. Consistent with Ahamed and Mallick (2019), we use our specification of equation (2) and include two interaction terms MM x HIGH and MM x LOW, where for MM we use both the dummy and categorical variables of bank involvement in Bank-MNO partnership (equation 3). Note that in equation (3) the interaction terms replace our variable MM. The intuition is to capture both effects of bank involvement in mobile money (the two measures) on their performance in 
high-deposit-funding on the one hand as opposed to low-deposit-funding banks on the other hand. Moreover, we also consider the level of bank diversification through the non-interest income and follow the same approach as presented above. We create two dummy variables, the first, HIGH diversified that takes the value one if the bank non-interest income is higher than the sample mean, and zero otherwise. The second, LOW diversified indicates that the bank non-interest income is lower than the sample mean and equals to one minus HIGH diversified.

$$
Y_{i t}=\lambda_{1} M M_{i t} \times H I G H_{i t}+\lambda_{2} M M_{i t} \times L O W_{i t}+\lambda_{3} H i g h_{i t}+\lambda_{4} X_{i t}+\eta_{i}+T_{t}+\omega_{i t}
$$

The results on banks with HIGH/LOW deposit funding are reported in Table 4A and 4B. We find that only the coefficients associated with LOW deposit funding are significant when MM is proxied by the dummy variable, and that the coefficients of the two interaction terms are different. Implying that mobile money improves profitability and efficiency of banks with low deposit funding. However, when considering the number of years of involvement in Bank-MNO partnership the results show a significant association between the coefficients of the two interaction terms and our measure of bank profitability, efficiency and stability, and that the coefficients of the two interaction terms are similar. Hence, number of years of Bank-MNO partnership since inception improves the performance of banks with HIGH or LOW deposit funding. Our findings suggest that deposit funding appear as a mechanism through which banks' involvement in mobile money affect their performance.

Taking our second possible channel, the level of bank diversification - non interest income - the results show that the coefficients associated with our two interaction terms HIGH/LOW diversified are all significant when considering the number of years of Bank-MNO partnership since inception. Moreover, the Wald test $\left(\lambda_{1}=\lambda_{2}\right)$ show that the coefficients of the interaction terms are similar. Thus, our results suggest that the level of diversification is a possible channel through which the number of years of banks are involved in the partnership improves their performance. 
Table 4A. The effect of deposit funding on bank performance in the EAC.

\begin{tabular}{|c|c|c|c|c|c|c|c|c|}
\hline \multirow{3}{*}{ Panel A } & \multicolumn{8}{|c|}{ ALL BANKS } \\
\hline & \multicolumn{4}{|c|}{ MM: Bank-MNO partnership ( 1 if yes, 0 otherwise) } & \multicolumn{4}{|c|}{ MM: Number of years of Bank-MNO partnership since inception } \\
\hline & $\begin{array}{l}\text { ROA } \\
\text { (1) }\end{array}$ & $\begin{array}{l}\text { ROE } \\
(2)\end{array}$ & $\begin{array}{l}\text { COST/INCOME } \\
\text { (3) }\end{array}$ & $\begin{array}{l}\text { Z-SCORE } \\
\text { (4) }\end{array}$ & $\begin{array}{l}\text { ROA } \\
(5)\end{array}$ & $\begin{array}{l}\text { ROE } \\
(6)\end{array}$ & $\begin{array}{l}\text { COST/INCOME } \\
\text { (7) }\end{array}$ & $\begin{array}{l}\text { Z-SCORE } \\
\text { (8) }\end{array}$ \\
\hline MM x High deposit funding $\left(\lambda_{1}\right)$ & $\begin{array}{l}-0.772 \\
(0.635)\end{array}$ & $\begin{array}{l}-3.900 \\
(5.058)\end{array}$ & $\begin{array}{l}5.466 \\
(10.373)\end{array}$ & $\begin{array}{l}1.236 \\
(2.174)\end{array}$ & $\begin{array}{l}0.203^{*} \\
(0.114)\end{array}$ & $\begin{array}{l}0.838 \\
(0.907)\end{array}$ & $\begin{array}{l}-4.581 * * \\
(1.826)\end{array}$ & $\begin{array}{l}0.908 * * \\
(0.384)\end{array}$ \\
\hline MM x Low deposit funding $\left(\lambda_{2}\right)$ & $\begin{array}{l}1.406 * * * \\
(0.485)\end{array}$ & $\begin{array}{l}8.816^{* *} \\
(3.866)\end{array}$ & $\begin{array}{l}-18.357^{* *} \\
(7.928)\end{array}$ & $\begin{array}{l}1.290 \\
(1.662)\end{array}$ & $\begin{array}{l}0.362^{* * * *} \\
(0.135)\end{array}$ & $\begin{array}{l}2.261^{* *} \\
(1.070)\end{array}$ & $\begin{array}{l}-8.576 * * * \\
(2.153)\end{array}$ & $\begin{array}{l}1.122^{* * *} \\
(0.453)\end{array}$ \\
\hline $\begin{array}{l}\text { F-statistics for test: }\left(\lambda_{1}=\lambda_{2}\right) \\
\text { p-value }\end{array}$ & $\begin{array}{l}9.32 \\
0.002\end{array}$ & $\begin{array}{l}5.00 \\
0.026\end{array}$ & $\begin{array}{l}4.17 \\
0.042\end{array}$ & $\begin{array}{l}0.00 \\
0.982\end{array}$ & $\begin{array}{l}1.20 \\
0.274\end{array}$ & $\begin{array}{l}1.51 \\
0.220\end{array}$ & $\begin{array}{l}2.94 \\
0.087\end{array}$ & $\begin{array}{l}0.19 \\
0.662\end{array}$ \\
\hline All controls included & YES & YES & YES & YES & YES & YES & YES & YES \\
\hline Observations & 461 & 461 & 461 & 461 & 461 & 461 & 461 & 461 \\
\hline R2 within & 0.414 & 0.345 & 0.387 & 0.065 & 0.408 & 0.341 & 0.406 & 0.086 \\
\hline $\mathrm{R} 2$ between & 0.239 & 0.289 & 0.266 & 0.024 & 0.248 & 0.298 & 0.260 & 0.017 \\
\hline Number of banks & 102 & 102 & 102 & 102 & 102 & 102 & 102 & 102 \\
\hline \multirow{3}{*}{ Panel B } & \multicolumn{4}{|c|}{ COMMERCIAL BANKS } & & & & \\
\hline & MM: Ban & INO partı & hip (1 if yes, 0 othe & & \multicolumn{4}{|c|}{ MM: Number of years of Bank-MNO partnership since inception } \\
\hline & $\begin{array}{l}\text { ROA } \\
\text { (1) }\end{array}$ & $\begin{array}{l}\text { ROE } \\
(2)\end{array}$ & $\begin{array}{l}\text { COST/INCOME } \\
\text { (3) }\end{array}$ & $\begin{array}{l}\text { Z-SCORE } \\
(4)\end{array}$ & $\begin{array}{l}\text { ROA } \\
(5)\end{array}$ & $\begin{array}{l}\text { ROE } \\
(6)\end{array}$ & $\begin{array}{l}\text { COST/INCOME } \\
\text { (7) }\end{array}$ & $\begin{array}{l}\text { Z-SCORE } \\
(8)\end{array}$ \\
\hline MM x High deposit funding $\left(\lambda_{1}\right)$ & $\begin{array}{l}-1.085 \\
(0.685)\end{array}$ & $\begin{array}{l}-6.696 \\
(5.299)\end{array}$ & $\begin{array}{l}9.067 \\
(10.987)\end{array}$ & $\begin{array}{l}2.572 \\
(2.308)\end{array}$ & $\begin{array}{l}0.185 \\
(0.131)\end{array}$ & $\begin{array}{l}0.849 \\
(1.011)\end{array}$ & $\begin{array}{l}-4.615^{* *} \\
(2.059)\end{array}$ & $\begin{array}{l}1.144 * * * \\
(0.431)\end{array}$ \\
\hline MM x Low deposit funding $\left(\lambda_{2}\right)$ & $\begin{array}{l}1.597 * * * \\
(0.606)\end{array}$ & $\begin{array}{l}8.224 * \\
(4.687)\end{array}$ & $\begin{array}{l}-21.010^{* *} \\
(9.718)\end{array}$ & $\begin{array}{l}2.252 \\
(2.042)\end{array}$ & $\begin{array}{l}0.350^{* * *} \\
(0.166)\end{array}$ & $\begin{array}{l}2.152^{*} \\
(1.281)\end{array}$ & $\begin{array}{l}-9.000 * * * \\
(2.608)\end{array}$ & $\begin{array}{l}1.523 * * * \\
(0.546)\end{array}$ \\
\hline $\begin{array}{l}\text { F-statistics for test: }\left(\lambda_{1}=\lambda_{2}\right) \\
\text { p-value }\end{array}$ & $\begin{array}{l}10.37 \\
0.001\end{array}$ & $\begin{array}{l}5.36 \\
0.021\end{array}$ & $\begin{array}{l}5.07 \\
0.025\end{array}$ & $\begin{array}{l}0.01 \\
0.909\end{array}$ & $\begin{array}{l}0.79 \\
0.375\end{array}$ & $\begin{array}{l}0.83 \\
0.363\end{array}$ & $\begin{array}{l}2.27 \\
0.133\end{array}$ & $\begin{array}{l}0.39 \\
0.534\end{array}$ \\
\hline All controls included & YES & YES & YES & YES & YES & YES & YES & YES \\
\hline Observations & 381 & 381 & 381 & 381 & 381 & 381 & 381 & 381 \\
\hline R2 within & 0.402 & 0.338 & 0.410 & 0.087 & 0.389 & 0.332 & 0.426 & 0.117 \\
\hline $\mathrm{R} 2$ between & 0.300 & 0.355 & 0.281 & 0.011 & 0.299 & 0.357 & 0.267 & 0.011 \\
\hline Number of banks & 84 & 84 & 84 & 84 & 84 & 84 & 84 & 84 \\
\hline
\end{tabular}

Number of banks

84

84

84

84

84

Note: Dependent variables: Return on assets (ROA), Return on equity (ROE), Cost to income ratio (COST/INCOME), and the sum of return on assets and equity ratio divided by the standard deviation of return on assets (Z-SCORE). MM, involvement in mobile money, stands alternatively for Bank-MNO partnership that equals 1 if yes, 0 otherwise, and the number of years of Bank-MNO partnership since inception. Robust standard errors are in brackets Controls included: HIGH is a dummy variable that takes the value 1 if banks has a deposit funding indicator that is higher than the sample mean, and 0 otherwise; SIZE is the logarithm of total asset; LLP is the ratio of loan loss provision over total assets; TL_TA is the ratio of total loans to total assets; ASSETS Growth is the growth rate of total assets; NII is the share of non-interest income over total operating income; OBS is the ratio of off-balance sheet over total assets; GDP Growth is the annual growth rate of gross domestic product; INFL is the annual growth rate of consumer price index. ***Significant at the $1 \%$ level, $* *$ Significant at the $5 \%$ level, *Significant at the $10 \%$ level. 
Table 4B. The effect of diversification on bank performance in the EAC.

\begin{tabular}{|c|c|c|c|c|c|c|c|c|}
\hline \multirow{3}{*}{ Panel A } & \multicolumn{8}{|c|}{ ALL BANKS } \\
\hline & \multicolumn{4}{|c|}{ MM: Bank-MNO partnership (1 if yes, 0 otherwise) } & \multicolumn{4}{|c|}{ MM: Number of years of Bank-MNO partnership since inception } \\
\hline & $\begin{array}{l}\text { ROA } \\
\text { (1) }\end{array}$ & $\begin{array}{l}\text { ROE } \\
\text { (2) }\end{array}$ & $\begin{array}{l}\text { COST/INCOME } \\
\text { (3) }\end{array}$ & $\begin{array}{l}\text { Z-SCORE } \\
\text { (4) }\end{array}$ & $\begin{array}{l}\text { ROA } \\
\text { (5) }\end{array}$ & $\begin{array}{l}\text { ROE } \\
(6)\end{array}$ & $\begin{array}{l}\text { COST/INCOME } \\
\text { (7) }\end{array}$ & $\begin{array}{l}\text { Z-SCORE } \\
\text { (8) }\end{array}$ \\
\hline MM x High diversified & $\begin{array}{l}0.806 \\
(0.558)\end{array}$ & $\begin{array}{l}4.033 \\
(4.365)\end{array}$ & $\begin{array}{l}-10.586 \\
(8.579)\end{array}$ & $\begin{array}{l}0.468 \\
(1.764)\end{array}$ & $\begin{array}{l}0.355^{* * *} \\
(0.135)\end{array}$ & $\begin{array}{l}1.714 \\
(1.058)\end{array}$ & $\begin{array}{l}-7.881 * * * \\
(2.048)\end{array}$ & $\begin{array}{l}0.960^{* *} \\
(0.425)\end{array}$ \\
\hline MM x Low diversified & $\begin{array}{l}0.303 \\
(0.483)\end{array}$ & $\begin{array}{l}2.285 \\
(3.776)\end{array}$ & $\begin{array}{l}-3.008 \\
(7.421)\end{array}$ & $\begin{array}{l}1.892 \\
(1.526)\end{array}$ & $\begin{array}{l}0.303^{* * *} * \\
(0.106)\end{array}$ & $\begin{array}{l}1.648^{* *} \\
(0.834)\end{array}$ & $\begin{array}{l}-5.441 * * * \\
(1.613)\end{array}$ & $\begin{array}{l}1.003 * * * \\
(0.335)\end{array}$ \\
\hline F-statistics for test & 0.99 & 0.20 & 0.95 & 0.80 & 0.26 & 0.01 & 2.51 & 0.02 \\
\hline p-value & 0.319 & 0.658 & 0.330 & 0.373 & 0.609 & 0.934 & 0.114 & 0.895 \\
\hline All controls included & YES & YES & YES & YES & YES & YES & YES & YES \\
\hline Observations & 466 & 466 & 466 & 466 & 466 & 466 & 466 & 466 \\
\hline R2 within & 0.314 & 0.261 & 0.367 & 0.083 & 0.327 & 0.268 & 0.392 & 0.102 \\
\hline $\mathrm{R} 2$ between & 0.227 & 0.264 & 0.238 & 0.018 & 0.229 & 0.267 & 0.233 & 0.023 \\
\hline Number of banks & 103 & 103 & 103 & 103 & 103 & 103 & 103 & 103 \\
\hline \multirow{3}{*}{ Panel B } & \multicolumn{8}{|c|}{ COMMERCIAL BANKS } \\
\hline & \multicolumn{4}{|c|}{ MM: Bank-MNO partnership (1 if yes, 0 otherwise) } & \multicolumn{4}{|c|}{ MM: Number of years of Bank-MNO partnership since inception } \\
\hline & $\begin{array}{l}\text { ROA } \\
\text { (1) }\end{array}$ & $\begin{array}{l}\text { ROE } \\
(2)\end{array}$ & $\begin{array}{l}\text { COST/INCOME } \\
\text { (3) }\end{array}$ & $\begin{array}{l}\text { Z-SCORE } \\
(4)\end{array}$ & $\begin{array}{l}\text { ROA } \\
\text { (5) }\end{array}$ & $\begin{array}{l}\text { ROE } \\
(6)\end{array}$ & $\begin{array}{l}\text { COST/INCOME } \\
\text { (7) }\end{array}$ & $\begin{array}{l}\text { Z-SCORE } \\
\text { (8) }\end{array}$ \\
\hline MM x High diversified & $\begin{array}{l}0.960 \\
(0.614)\end{array}$ & $\begin{array}{l}3.406 \\
(4.706)\end{array}$ & $\begin{array}{l}-12.191 \\
(9.855)\end{array}$ & $\begin{array}{l}1.575 \\
(2.028)\end{array}$ & $\begin{array}{l}0.317 * * \\
(0.139)\end{array}$ & $\begin{array}{l}1.495 \\
(1.067)\end{array}$ & $\begin{array}{l}-8.094 * * * \\
(2.197)\end{array}$ & $\begin{array}{l}1.206^{* * * *} \\
(0.454)\end{array}$ \\
\hline MM x Low diversified & $\begin{array}{l}-0.110 \\
(0.523)\end{array}$ & $\begin{array}{l}-1.150 \\
(4.009)\end{array}$ & $\begin{array}{l}0.996 \\
(8.397)\end{array}$ & $\begin{array}{l}3.028^{*} \\
(1.728)\end{array}$ & $\begin{array}{l}0.238^{* * *} \\
(0.117)\end{array}$ & $\begin{array}{l}1.310 \\
(0.896)\end{array}$ & $\begin{array}{l}-5.486^{* * * *} \\
(1.844)\end{array}$ & $\begin{array}{l}1.313 * * * \\
(0.381)\end{array}$ \\
\hline F-statistics for test & 3.98 & 1.23 & 2.34 & 0.67 & 0.59 & 0.05 & 2.60 & 0.10 \\
\hline p-value & 0.047 & 0.269 & 0.127 & 0.413 & 0.443 & 0.815 & 0.108 & 0.749 \\
\hline All controls included & YES & YES & YES & YES & YES & YES & YES & YES \\
\hline Observations & 381 & 381 & 381 & 381 & 381 & 381 & 381 & 381 \\
\hline R2 within & 0.380 & 0.326 & 0.387 & 0.089 & 0.383 & 0.329 & 0.411 & 0.117 \\
\hline $\mathrm{R} 2$ between & 0.284 & 0.336 & 0.257 & 0.006 & 0.280 & 0.332 & 0.247 & 0.006 \\
\hline Number of banks & 84 & 84 & 84 & 84 & 84 & 84 & 84 & 84 \\
\hline
\end{tabular}

Note: Dependent variables: Return on assets (ROA), Return on equity (ROE), Cost to income ratio (COST/INCOME), and the sum of return on assets and equity ratio divided by the standard deviation of return on assets (Z-SCORE). MM, involvement in mobile money, stands alternatively for Bank-MNO partnership that equals 1 if yes, 0 otherwise, and the number of years of Bank-MNO partnership since inception. Robust standard errors are in brackets Controls included: HIGH is a dummy variable that takes the value 1 if banks has a non-interest income indicator that is higher than the sample mean, and 0 otherwise; SIZE is the logarithm of total asset; LLP is the ratio of loan loss provision over total assets; TL TA is the ratio of total loans to total assets; ASSETS Growth is the growth rate of total assets; NII is the share of non-interest income over total operating income; OBS is the ratio of off-balance sheet over total assets; GDP Growth is the annual growth rate of gross domestic product; INFL is the annual growth rate of consumer price index. ***Significant at the $1 \%$ level, **Significant at the $5 \%$ level, *Significant at the $10 \%$ level. 


\section{Conclusion}

In this study, our main objective is to analyse the possible effect of mobile money adoption by banks on their performance using a sample of 170 financial institutions from the five partner states of the East African Community (EAC), a worldwide leading region in mobile money adoption and usage. This financial technology virtually accessible to anyone with a mobile phone holds the promise to "leapfrog" the provision of banking services in developing countries thanks to the comparatively high penetration of mobile phones in contrast to the low density of banking infrastructure. It allows banks to leverage innovative and less costly business models to serve unbanked or underbanked people by overcoming existing market inefficiencies and reducing the prohibitive cost of maintaining physical bank branches in rural locations. There is a paucity of literature examining the impact of mobile money on firms and, to the best of our knowledge, there is no empirical evidence about whether mobile money hinders or promotes bank performance. Our paper aims at filling this gap by using a hand collected data sample obtained by screening financial institutions' websites and GSMA tracker to identify those that are involved in mobile money schemes. We supplement our data with bank specific data from Bankscope, macroeconomic data from World Development Indicators, and the number of mobile money users and the value of mobile money transactions from the Financial Access Survey (IMF).

Our main findings indicate that the number of years of Bank-MNO partnership since inception is strongly related to bank performance (profitability, efficiency or stability) while there are no significant effects when the bank's status of involvement at a point in time is considered. Restricting our sample to commercial banks yields similar results although there is a drop-in significance when looking at bank profitability. Moreover, we split our sample into small and large banks and find that results on large banks perfectly mimic those on the banking sector. For small banks, the results show that being involved in mobile money is strongly associated with both their profitability and efficiency irrespective of their degree of involvement in a partnership with an MNO, but no significant association with stability. In further investigations, we check the consistency of our results using six alternative measures of our dependent variables, considering one-year lagged variable and country-level measures of mobile money adoption as alternatives of our independent variable of interest (MM). We also extend our analysis to all financial institutions operating in the EAC. Overall, we find that our main conclusions are unaltered. Exploring channels 
through which mobile money affects bank performance, our findings suggest that improved access to retail deposits and income diversification are possible candidates.

Our study has important policy implications and contributes to the debate of the capacity of mobile money system to galvanize the banking sector by enhancing stability and promoting financial inclusion. The findings provide novel insights concerning the convenience of mobile money development for the banking sector. More specifically, our results contribute to the debate about the benefit of mobile money innovation on bank performance. Nevertheless, while our paper points to efficiency, profitability and stability gains that accrue to mobile money adopters (the bright side), it is critical to bear in mind potential vulnerabilities (dark side) that could result in intensified competition once this innovation matures. For instance, issues of late repayment and default or debt cycling and over-indebtedness have started to emerge following the rapid expansion of digital loans. Moreover, evidence from the developed world shows how new technologies often bring new risks. The case of securitization that went from being a well-acclaimed financial innovation to becoming one of the root causes of the Global Financial Crisis of 2008 is a vivid illustration. Given the perennial concern that regulation always lags behind innovation, it is of high importance that future research considers such potential risks. 


\section{References}

Ahamed, M.M., Mallick, S.K., 2019. Is financial inclusion good for bank stability? International evidence. J. Econ. Behav. Organ. 157, 403-427. https://doi.org/10.1016/j.jebo.2017.07.027

Anagnostopoulos, I., 2018. Fintech and regtech: Impact on regulators and banks. J. Econ. Bus. 100, 7-25. https://doi.org/10.1016/j.jeconbus.2018.07.003

Aron, J., 2017. ' Leapfrogging ': a Survey of the Nature and Economic Implications of Mobile Money. CSAE Work. Pap. WPS/2017-02.

Basel Committee on Banking Supervision, 2018. Sound Practices: Implications of fintech developments for banks and bank supervisors. Bank Int. Settlements.

Beccalli, E., 2007. Does IT investment improve bank performance? Evidence from Europe. J. Bank. Financ. 31, 2205-2230.

Beck, T., Chen, T., Lin, C., Song, F.M., 2016. Financial innovation: The bright and the dark sides. J. Bank. Financ. 72, 28-51. https://doi.org/10.1016/j.jbankfin.2016.06.012

Berger, A.N., Hasan, I., Zhou, M., 2010. The effects of focus versus diversification on bank performance: Evidence from Chinese banks. J. Bank. Financ. 34, 1417-1435. https://doi.org/10.1016/j.jbankfin.2010.01.010

BFA, 2017. East Africa Mobile Money Cross-Border Payments : Market demand Side. Consult. Gr. to Assist Poor 1-92.

Bharadwaj, P., Jack, W., Suri, T., 2019. Fintech and Household Resilience to Shocks: Evidence from Digital Loans in Kenya. Cambridge, MA. https://doi.org/10.3386/w25604

Bitar, M., Pukthuanthong, K., Walker, T., 2018. The effect of capital ratios on the risk , efficiency and profitability of banks : Evidence from OECD countries. J. Int. Financ. Mark. Institutions Money 53, 227-262. https://doi.org/10.1016/j.intfin.2017.12.002

Cook, W., McKay, C., 2017. Banking in the M-PESA Age: Lessons from Kenya. Work. Pap. Washington, D.C. CGAP.

Demirgüç-Kunt, A., Huizinga, H., 2010. Bank activity and funding strategies: The impact on risk and returns. J. financ. econ. 98, 626-650. https://doi.org/10.1016/j.jfineco.2010.06.004

Dos Santos, B.L., Peffers, K., 1995. No TitleRewards to Investors in Innovative Information Technology Applications: First Movers and Early Followers in ATMs. Organ. Sci. 6, 241259.

Drasch, B.J., Schweizer, A., Urbach, N., 2018. Integrating the 'Troublemakers': A taxonomy for cooperation between banks and fintechs. J. Econ. Bus. 100, 26-42. https://doi.org/10.1016/j.jeconbus.2018.04.002

Flaming, M., Mitha, A., Hanouch, M., Zetterli, P., Bull, G., 2013. Partnerships in Mobile Financial Services : Factors for Success. Int. Financ. Corp. World Bank Gr.

Frame, W.S., Wall, L., White, L.J., 2018. Technological Change and Financial Innovation in Banking: Some Implications for Fintech. Fed. Reserv. Bank Atlanta, Work. Pap. No. 201811. https://doi.org/10.29338/wp2018-11

Frame, W.S., White, L.J., 2004. Empirical studies of financial innovation: lots of talk, little action?, Journal of Economic Literature. https://doi.org/10.1111/j.1467629X.1984.tb00054.X

Fuentelsaz, L., Gómez, J., Palomas, S., 2012. Production technologies and financial performance : The effect of uneven diffusion among competitors. Res. Policy 41, 401-413. https://doi.org/10.1016/j.respol.2011.09.006

Fuentelsaz, L., Gómez, J., Palomas, S., 2009. The effects of new technologies on productivity: An intrafirm diffusion-based assessment. Res. Policy 38, 1172-1180. 
https://doi.org/10.1016/j.respol.2009.04.003

Fuentelsaz, L., Gomez, J., Polo, Y., 2003. Intrafirm diffusion of new technologies : an empirical application 32, 533-551.

Greenacre, J., Buckley, R.P., 2014. Using Trusts to Protect Mobile Money Customers. Singapore J. Leg. Stud. 59-78.

Han, R., Melecky, M., 2014. Financial Inclusion for Financial Stability: Access to Bank Deposits and the Growth of Deposits in the Global Financial Crisis. Policy Res. Work. Pap. 6577, World Bank. https://doi.org/10.1596/1813-9450-6577

Hirt, M., Willmott, P., 2014. Strategic principles for competing in the digital age. McKinsey Q. $1-13$.

International Monetary Fund, 2018. The Bali Fintech Agenda, IMF Policy Paper, Washington, DC.

Jack, W., Suri, T., 2016. The long-run poverty and gender impacts of mobile money. Science (80. ). 1288-1292. https://doi.org/10.1126/science.aah5309

Jack, W., Suri, T., 2014. Risk sharing and transactions costs: Evidence from Kenya's mobile money revolution. Am. Econ. Rev. 104, 183-223. https://doi.org/10.1257/aer.104.1.183

Jagtiani, J., John, K., 2018. Fintech: The Impact on Consumers and Regulatory Responses. J. Econ. Bus. 100, 1-6. https://doi.org/10.1016/j.jeconbus.2018.11.002

Jagtiani, J., Lemieux, C., 2018. Do fintech lenders penetrate areas that are underserved by traditional banks? J. Econ. Bus. 100, 43-54. https://doi.org/10.1016/j.jeconbus.2018.03.001

Karshenas, M., Stoneman, P.L., 1993. Rank, Stock, Order, and Epidemic Effects in the Diffusion of New Process Technologies: An Empirical Model. RAND J. Econ. 24, 503-528. https://doi.org/10.2307/2555742

Kirilenko, A.A., Lo, A.W., 2013. Moore's Law vs. Murphy's Law: Algorithmic Trading and Its Discontents. J. Econ. Perspect. 27, 51-72. https://doi.org/10.2139/ssrn.2235963

Koami, K.A., Afrika, J.-G., Akanni-Honvo, A., Tomen, H.N., Abimbola, O., Davis, W., 2016. Africa Regional Integration Index: Report 2016. African Union Comm. African Dev. Bank, Econ. Comm. Africa 1-52.

Köster, H., Pelster, M., 2017. Financial penalties and bank performance. J. Bank. Financ. 79, 5773. https://doi.org/10.1016/j.jbankfin.2017.02.009

Lashitew, A.A., Tulder, R. van, Liasse, Y., 2019. Mobile phones for financial inclusion: What explains the diffusion of mobile money innovations? Res. Policy 48, 1201-1215.

Lepetit, L., Rugemintwari, C., Strobel, F., 2015. Monetary, Financial and Fiscal Stability in the East African Community: Ready for a Monetary Union? World Econ. 38, 1179-1204. https://doi.org/10.1111/twec.12243

Liang, H.Y., Ching, Y.P., Chan, K.C., 2013. Enhancing bank performance through branches or representative offices? Evidence from European banks. Int. Bus. Rev. 22, 495-508. https://doi.org/10.1016/j.ibusrev.2012.07.003

Mas, I., Ng'weno, A., 2010. Three keys to M-PESA's success: Branding, channel management and pricing. J. Payments Strateg. Syst. 4.

Mbiti, I., David Weil, 2016. Mobile Banking: the Impact of M-Pesa in Kenya. African Successes, Vol. III Mod. Dev. Edwards, Johnson, Weil. https://doi.org/10.1007/s13398-014-0173-7.2

Meslier, C., Tacneng, R., Tarazi, A., 2014. Is bank income diversification beneficial? Evidence from an emerging economy. J. Int. Financ. Mark. Institutions Money 31, 97-126. https://doi.org/10.1016/j.intfin.2014.03.007

Minischetti, E., Scharwatt, C., 2016. Global adoption of mobile money in 2015: A look at the data [WWW Document]. GSMA, Mob. Dev. URL 
https://www.gsma.com/mobilefordevelopment/programme/mobile-money/global-adoptionmobile-money-2015-look-data/

Mostak Ahamed, M., 2017. Asset quality, non-interest income, and bank profitability: Evidence from Indian banks. Econ. Model. 63, 1-14. https://doi.org/10.1016/j.econmod.2017.01.016

Munyegera, G.K., Matsumoto, T., 2016. Mobile Money, Remittances, and Household Welfare: Panel Evidence from Rural Uganda. World Dev. 79, 127-137. https://doi.org/10.1016/j.worlddev.2015.11.006

Philippon, T., 2018. The FinTech Opportunity. Work. Pap. https://doi.org/10.3386/w22476

Saghi-Zedek, N., 2016. Product diversification and bank performance: Does ownership structure matter? J. Bank. Financ. 71, 154-167.

Sanya, S., Gaertner, M., 2012. Assessing Bank Competition within the East African Community. IMF Work. Pap. 12, 1. https://doi.org/10.5089/9781463933173.001

Scott, S. V., Reenen, J. Van, Zachariadis, M., 2017. The long-term effect of digital innovation on bank performance: An empirical study of SWIFT adoption in financial services. Res. Policy 46, 984-1004. https://doi.org/10.1016/j.respol.2017.03.010

Shaban, M., James, G.A., 2014. The effects of ownership change on bank performance and risk exposure: Evidence from indonesia. J. Bank. Financ. 0, 1-15. https://doi.org/10.1016/j.jbankfin.2017.02.002

Stijns, J.-P., Revoltella, D., 2016. Banking in sub-Saharan Africa Recent Trends and Digital Financial Inclusion, European Investment Bank.

Sy, A.N.R., Maino, R., Massara, A., Perez-Saiz, H., Sharma, P., 2019. FinTech in Sub-Saharan African Countries: A Game Changer? IMF, Dep. Pap. No. 19/04.

Thakor, A. V., 2019. Fintech and Banking. Available at SSRN: https//ssrn.com/abstract=3332550 or http//dx.doi.org/10.2139/ssrn.3332550.

The Economist Unit Intelligence, 2015. The disruption of banking.

The Financial Stability Board, 2017. Financial Stability Implications from Fintech: Supervisory and Regulatory Issues that Merit Authorities' Attention.

The World Bank, 2019. World Development Indicators, Washington, DC.

United Nations Conference on Trade and Development, 2012. Mobile Money for Business Development in East African Community. United Nations Publ.

Van Der Boor, P., Oliveira, P., Veloso, F., 2014. Users as innovators in developing countries: The global sources of innovation and diffusion in mobile banking services. Res. Policy 43, 1594-1607. https://doi.org/10.1016/j.respol.2014.05.003

World Bank Group, 2018. The Little Data Book on Financial Inclusion. https://doi.org/10.1596/978-1-4648-0552-3 


\section{Appendix}

Table A.1A. Mobile money and bank performance in the East African Community

\begin{tabular}{|c|c|c|c|c|c|c|c|c|c|c|c|c|}
\hline \multirow[b]{3}{*}{ Panel A } & \multicolumn{12}{|c|}{ ALL BANKS } \\
\hline & \multicolumn{6}{|c|}{ Bank-MNO partnership (1 if yes, 0 otherwise) } & \multicolumn{6}{|c|}{ Number of years of Bank-MNO partnership since inception } \\
\hline & $\begin{array}{l}\text { RAROA } \\
\text { (1) }\end{array}$ & $\begin{array}{l}\text { NIM } \\
\text { (2) }\end{array}$ & $\begin{array}{l}\text { NIEAA } \\
\text { (3) }\end{array}$ & $\begin{array}{l}\text { NOITAA } \\
\text { (4) }\end{array}$ & $\begin{array}{l}\text { Z-SCORE } \\
(3 y) \\
(5)\end{array}$ & $\begin{array}{l}\text { Z-SCORE } \\
(5 y) \\
(6)\end{array}$ & $\begin{array}{l}\text { RAROA } \\
\text { (7) }\end{array}$ & $\begin{array}{l}\text { NIM } \\
\text { (8) }\end{array}$ & $\begin{array}{l}\text { NIEAA } \\
\text { (9) }\end{array}$ & $\begin{array}{l}\text { NOITAA } \\
\text { (10) }\end{array}$ & $\begin{array}{l}\text { Z-SCORE } \\
(3 y) \\
(11)\end{array}$ & $\begin{array}{l}\text { Z-SCORE } \\
(5 y) \\
(12)\end{array}$ \\
\hline MM & $\begin{array}{l}0.142 \\
(0.101)\end{array}$ & $\begin{array}{l}0.267 \\
(0.261)\end{array}$ & $\begin{array}{l}0.051 \\
(0.416)\end{array}$ & $\begin{array}{l}0.175 \\
(0.233)\end{array}$ & $\begin{array}{l}0.141 \\
(0.101)\end{array}$ & $\begin{array}{l}0.142 \\
(0.099)\end{array}$ & $\begin{array}{l}0.077 * * * \\
(0.023)\end{array}$ & $\begin{array}{l}0.215 * * * \\
(0.060)\end{array}$ & $\begin{array}{l}-0.217 * * \\
(0.097)\end{array}$ & $\begin{array}{l}-0.166 \text { *** } \\
(0.054)\end{array}$ & $\begin{array}{l}0.078 * * * \\
(0.023)\end{array}$ & $\begin{array}{l}0.079 * * * \\
(0.023)\end{array}$ \\
\hline $\begin{array}{l}\text { Controls } \\
\text { included }\end{array}$ & YES & YES & YES & YES & YES & YES & YES & YES & YES & YES & YES & YES \\
\hline Observations & 466 & 466 & 466 & 453 & 466 & 466 & 466 & 466 & 466 & 453 & 466 & 466 \\
\hline R2 within & 0.304 & 0.571 & 0.737 & 0.369 & 0.303 & 0.312 & 0.322 & 0.585 & 0.740 & 0.386 & 0.320 & 0.330 \\
\hline R2 between & 0.254 & 0.253 & 0.343 & 0.369 & 0.242 & 0.242 & 0.254 & 0.351 & 0.332 & 0.365 & 0.242 & 0.242 \\
\hline Number of banks & 103 & 103 & 103 & 102 & 103 & 103 & 103 & 103 & 103 & 102 & 103 & 103 \\
\hline Fixed effects & YES & YES & YES & YES & YES & YES & YES & YES & YES & YES & YES & YES \\
\hline Time dummies & YES & YES & YES & YES & YES & YES & YES & YES & YES & YES & YES & YES \\
\hline \multirow[b]{3}{*}{ Panel $B$} & \multicolumn{12}{|c|}{ COMMERCIAL BANKS } \\
\hline & \multicolumn{6}{|c|}{ Bank-MNO partnership (1 if yes, 0 otherwise) } & \multicolumn{6}{|c|}{ Number of years of Bank-MNO partnership since inception } \\
\hline & $\begin{array}{l}\text { RAROA } \\
\text { (1) }\end{array}$ & $\begin{array}{l}\text { NIM } \\
(2) \\
\end{array}$ & $\begin{array}{l}\text { NIEAA } \\
\text { (3) }\end{array}$ & $\begin{array}{l}\text { NOITAA } \\
\text { (4) }\end{array}$ & $\begin{array}{l}\text { Z-SCORE } \\
(3 y) \\
(5) \\
\end{array}$ & $\begin{array}{l}\text { Z-SCORE } \\
(5 y) \\
(6) \\
\end{array}$ & $\begin{array}{l}\text { RAROA } \\
(7) \\
\end{array}$ & $\begin{array}{l}\text { NIM } \\
(8) \\
\end{array}$ & $\begin{array}{l}\text { NIEAA } \\
(9) \\
\end{array}$ & $\begin{array}{l}\text { NOITAA } \\
\text { (10) }\end{array}$ & $\begin{array}{l}\text { Z-SCORE } \\
(3 y) \\
(11) \\
\end{array}$ & $\begin{array}{l}\text { Z-SCORE } \\
(5 y) \\
(12) \\
\end{array}$ \\
\hline MM & $\begin{array}{l}0.065 \\
(0.110)\end{array}$ & $\begin{array}{l}0.137 \\
(0.230)\end{array}$ & $\begin{array}{l}0.180 \\
(0.497)\end{array}$ & $\begin{array}{l}0.160 \\
(0.276)\end{array}$ & $\begin{array}{l}0.066 \\
(0.111)\end{array}$ & $\begin{array}{l}0.073 \\
(0.108)\end{array}$ & $\begin{array}{l}0.061 * * \\
(0.025)\end{array}$ & $\begin{array}{l}0.162 * * * * \\
(0.052)\end{array}$ & $\begin{array}{l}-0.215^{*} \\
(0.114)\end{array}$ & $\begin{array}{l}-0.169 * * * \\
(0.063)\end{array}$ & $\begin{array}{l}0.062 * * \\
(0.025)\end{array}$ & $\begin{array}{l}0.063^{* *} \\
(0.025)\end{array}$ \\
\hline $\begin{array}{l}\text { Controls } \\
\text { included } \\
\end{array}$ & YES & YES & YES & YES & YES & YES & YES & YES & YES & YES & YES & YES \\
\hline Observations & 381 & 381 & 381 & 372 & 381 & 381 & 381 & 381 & 381 & 372 & 381 & 381 \\
\hline R2 within & 0.366 & 0.462 & 0.697 & 0.393 & 0.363 & 0.369 & 0.378 & 0.479 & 0.700 & 0.408 & 0.376 & 0.383 \\
\hline R2 between & 0.296 & 0.0203 & 0.276 & 0.473 & 0.289 & 0.290 & 0.290 & 0.0562 & 0.265 & 0.466 & 0.284 & 0.284 \\
\hline Number of banks & 84 & 84 & 84 & 83 & 84 & 84 & 84 & 84 & 84 & 83 & 84 & 84 \\
\hline Fixed effects & YES & YES & YES & YES & YES & YES & YES & YES & YES & YES & YES & YES \\
\hline Time dummies & YES & YES & YES & YES & YES & YES & YES & YES & YES & YES & YES & YES \\
\hline
\end{tabular}


Table A.1B. Mobile money and bank performance in the East African Community (Small vs. Large banks).

\begin{tabular}{|c|c|c|c|c|c|c|c|c|c|c|c|c|}
\hline \multirow[b]{3}{*}{ Panel A } & \multicolumn{12}{|c|}{ ALL BANKS } \\
\hline & \multicolumn{6}{|c|}{ Bank-MNO partnership (1 if yes, 0 otherwise) } & \multicolumn{6}{|c|}{ Number of years of Bank-MNO partnership since inception } \\
\hline & $\begin{array}{l}\text { RAROA } \\
\text { (1) }\end{array}$ & $\begin{array}{l}\text { NIM } \\
(2)\end{array}$ & $\begin{array}{l}\text { NIEAA } \\
\text { (3) }\end{array}$ & $\begin{array}{l}\text { NOITAA } \\
\text { (4) }\end{array}$ & $\begin{array}{l}\text { Z-SCORE (3y) } \\
\text { (5) }\end{array}$ & $\begin{array}{l}\text { Z-SCORE } \\
(5 y) \\
(6)\end{array}$ & $\begin{array}{l}\text { RAROA } \\
\text { (7) }\end{array}$ & $\begin{array}{l}\text { NIM } \\
\text { (8) }\end{array}$ & $\begin{array}{l}\text { NIEAA } \\
\text { (9) }\end{array}$ & $\begin{array}{l}\text { NOITAA } \\
\text { (10) }\end{array}$ & $\begin{array}{l}\text { Z-SCORE (3y) } \\
\text { (11) }\end{array}$ & $\begin{array}{l}\text { Z-SCORE (5y) } \\
\text { (12) }\end{array}$ \\
\hline MM & $\begin{array}{l}0.006 \\
(0.113)\end{array}$ & $\begin{array}{l}0.078 \\
(0.294)\end{array}$ & $\begin{array}{l}0.315 \\
(0.477)\end{array}$ & $\begin{array}{l}0.185 \\
(0.265)\end{array}$ & $\begin{array}{l}0.007 \\
(0.114)\end{array}$ & $\begin{array}{l}0.014 \\
(0.112)\end{array}$ & $\begin{array}{l}0.065^{* * * *} \\
(0.023)\end{array}$ & $\begin{array}{l}0.186^{* * * *} \\
(0.060)\end{array}$ & $\begin{array}{l}-0.189^{*} \\
(0.098)\end{array}$ & $\begin{array}{l}-0.156^{* * * *} \\
(0.054)\end{array}$ & $\begin{array}{l}0.066 * * * \\
(0.023)\end{array}$ & $\begin{array}{l}0.067 * * * \\
(0.023)\end{array}$ \\
\hline SMALL & $\begin{array}{l}0.166^{* *} \\
(0.078)\end{array}$ & $\begin{array}{l}0.603 * * * \\
(0.203)\end{array}$ & $\begin{array}{l}-0.281 \\
(0.329)\end{array}$ & $\begin{array}{l}-0.051 \\
(0.187)\end{array}$ & $\begin{array}{l}0.168 * * \\
(0.078)\end{array}$ & $\begin{array}{l}0.163 * * \\
(0.077)\end{array}$ & $\begin{array}{l}0.149^{*} \\
(0.077)\end{array}$ & $\begin{array}{l}0.538 * * * \\
(0.200)\end{array}$ & $\begin{array}{l}-0.146 \\
(0.326)\end{array}$ & $\begin{array}{l}0.133 \\
(0.183)\end{array}$ & $\begin{array}{l}0.151^{*} \\
(0.078)\end{array}$ & $\begin{array}{l}0.145^{*} \\
(0.076)\end{array}$ \\
\hline MM x SMALL & $\begin{array}{l}0.337 * * \\
(0.143)\end{array}$ & $\begin{array}{l}0.449 \\
(0.372)\end{array}$ & $\begin{array}{l}-0.660 \\
(0.602)\end{array}$ & $\begin{array}{l}-0.021 \\
(0.338)\end{array}$ & $\begin{array}{l}0.332 * * \\
(0.144)\end{array}$ & $\begin{array}{l}0.318^{* *} \\
(0.141)\end{array}$ & $\begin{array}{l}0.101^{* *} \\
(0.046)\end{array}$ & $\begin{array}{l}0.149 \\
(0.118)\end{array}$ & $\begin{array}{l}-0.339^{*} \\
(0.192)\end{array}$ & $\begin{array}{l}-0.252 * * \\
(0.108)\end{array}$ & $\begin{array}{l}0.099 * * \\
(0.046)\end{array}$ & $\begin{array}{l}0.095^{* *} \\
(0.045)\end{array}$ \\
\hline Total effect & $\begin{array}{l}0.503 * * * \\
(0.140)\end{array}$ & $\begin{array}{l}1.052 * * * \\
(0.363)\end{array}$ & $\begin{array}{l}-0.941 \\
(0.588)\end{array}$ & $\begin{array}{l}-0.071 \\
(0.331)\end{array}$ & $\begin{array}{l}0.501 * * * \\
(0.140)\end{array}$ & $\begin{array}{l}0.481 * * * \\
(0.138)\end{array}$ & $\begin{array}{l}0.250 * * * \\
(0.077)\end{array}$ & $\begin{array}{l}0.687 * * * \\
(0.199)\end{array}$ & $\begin{array}{l}-0.485 \\
(0.325)\end{array}$ & $\begin{array}{l}-0.119 \\
(0.182)\end{array}$ & $\begin{array}{l}0.251 * * * \\
(0.077)\end{array}$ & $\begin{array}{l}0.241 * * * \\
(0.076)\end{array}$ \\
\hline Controls included & YES & YES & YES & YES & YES & YES & YES & YES & YES & YES & YES & YES \\
\hline Observations & 466 & 466 & 466 & 453 & 466 & 466 & 466 & 466 & 466 & 453 & 466 & 466 \\
\hline R2 within & 0.333 & 0.588 & 0.739 & 0.369 & 0.331 & 0.338 & 0.345 & 0.599 & 0.744 & 0.395 & 0.343 & 0.352 \\
\hline R2 between & 0.240 & 0.221 & 0.343 & 0.368 & 0.228 & 0.228 & 0.245 & 0.308 & 0.329 & 0.368 & 0.232 & 0.232 \\
\hline Number of banks & 103 & 103 & 103 & 102 & 103 & 103 & 103 & 103 & 103 & 102 & 103 & 103 \\
\hline Fixed effects & YES & YES & YES & YES & YES & YES & YES & YES & YES & YES & YES & YES \\
\hline \multirow[t]{3}{*}{ Time dummies } & YES & YES & YES & YES & YES & YES & YES & YES & YES & YES & YES & YES \\
\hline & \multicolumn{12}{|c|}{ COMMERCIAL BANKS } \\
\hline & \multicolumn{6}{|c|}{ Bank-MNO partnership (1 if yes, 0 otherwise) } & \multicolumn{6}{|c|}{ Number of years of Bank-MNO partnership since inception } \\
\hline Panel B & $\begin{array}{l}\text { RAROA } \\
\text { (1) }\end{array}$ & NIM & $\begin{array}{l}\text { NIEAA } \\
\text { (3) }\end{array}$ & $\begin{array}{l}\text { NOITAA } \\
\text { (4) }\end{array}$ & $\begin{array}{l}\text { Z-SCORE (3y) } \\
\text { (5) }\end{array}$ & $\begin{array}{l}\text { Z-SCORE } \\
(5 y) \\
(6)\end{array}$ & $\begin{array}{l}\text { RAROA } \\
\text { (7) }\end{array}$ & $\begin{array}{l}\text { NIM } \\
\text { (8) }\end{array}$ & $\begin{array}{l}\text { NIEAA } \\
\text { (9) }\end{array}$ & $\begin{array}{l}\text { NOITAA } \\
\text { (10) }\end{array}$ & $\begin{array}{l}\text { Z-SCORE (3y) } \\
\text { (11) }\end{array}$ & $\begin{array}{l}\text { Z-SCORE (5y) } \\
\text { (12) }\end{array}$ \\
\hline MM & $\begin{array}{l}-0.095 \\
(0.119)\end{array}$ & $\begin{array}{l}-0.125 \\
(0.252)\end{array}$ & $\begin{array}{l}0.490 \\
(0.551)\end{array}$ & $\begin{array}{l}0.127 \\
(0.304)\end{array}$ & $\begin{array}{l}-0.092 \\
(0.120)\end{array}$ & $\begin{array}{l}-0.080 \\
(0.117)\end{array}$ & $\begin{array}{l}0.050 * * \\
(0.025)\end{array}$ & $\begin{array}{l}0.140 * * * \\
(0.052)\end{array}$ & $\begin{array}{l}-0.187 \\
(0.115)\end{array}$ & $\begin{array}{l}-0.166 * * * \\
(0.063)\end{array}$ & $\begin{array}{l}0.051 * * \\
(0.025)\end{array}$ & $\begin{array}{l}0.053 * * \\
(0.025)\end{array}$ \\
\hline SMALL & $\begin{array}{l}0.170 * * \\
(0.083)\end{array}$ & $\begin{array}{l}0.379 * * \\
(0.174)\end{array}$ & $\begin{array}{l}-0.464 \\
(0.382)\end{array}$ & $\begin{array}{l}-0.119 \\
(0.213)\end{array}$ & $\begin{array}{l}0.173 * * \\
(0.083)\end{array}$ & $\begin{array}{l}0.166^{* * *} \\
(0.081)\end{array}$ & $\begin{array}{l}0.159^{*} \\
(0.083)\end{array}$ & $\begin{array}{l}0.331^{*} \\
(0.174)\end{array}$ & $\begin{array}{l}-0.305 \\
(0.382)\end{array}$ & $\begin{array}{l}0.136 \\
(0.210)\end{array}$ & $\begin{array}{l}0.161^{*} \\
(0.083)\end{array}$ & $\begin{array}{l}0.154 * \\
(0.081)\end{array}$ \\
\hline MM x SMALL & $\begin{array}{l}0.392 * * \\
(0.154)\end{array}$ & $\begin{array}{l}0.605^{*} \\
(0.325)\end{array}$ & $\begin{array}{l}-0.711 \\
(0.711)\end{array}$ & $\begin{array}{l}0.156 \\
(0.398)\end{array}$ & $\begin{array}{l}0.387 * * \\
(0.155)\end{array}$ & $\begin{array}{l}0.376^{* * *} \\
(0.151)\end{array}$ & $\begin{array}{l}0.109 * * \\
(0.052)\end{array}$ & $\begin{array}{l}0.182^{*} \\
(0.110)\end{array}$ & $\begin{array}{l}-0.363 \\
(0.241)\end{array}$ & $\begin{array}{l}-0.328 * * \\
(0.136)\end{array}$ & $\begin{array}{l}0.108 * * \\
(0.053)\end{array}$ & $\begin{array}{l}0.105^{* *} \\
(0.051)\end{array}$ \\
\hline Total effect & $\begin{array}{l}0.562 * * * \\
(0.154)\end{array}$ & $\begin{array}{l}0.984 * * * \\
(0.324)\end{array}$ & $\begin{array}{l}-1.175^{*} \\
(0.709)\end{array}$ & $\begin{array}{l}0.037 \\
(0.397)\end{array}$ & $\begin{array}{l}0.560 * * * \\
(0.154)\end{array}$ & $\begin{array}{l}0.542 \text { *** } \\
(0.151)\end{array}$ & $\begin{array}{l}0.268 * * * \\
(0.084)\end{array}$ & $\begin{array}{l}0.513 \text { *** } \\
(0.177)\end{array}$ & $\begin{array}{l}-0.668^{*} \\
(0.389)\end{array}$ & $\begin{array}{l}-0.192 \\
(0.214)\end{array}$ & $\begin{array}{l}0.270 * * * \\
(0.085)\end{array}$ & $\begin{array}{l}0.259 * * * \\
(0.083)\end{array}$ \\
\hline Controls included & YES & YES & YES & YES & YES & YES & YES & YES & YES & YES & YES & YES \\
\hline Observations & 381 & 381 & 381 & 372 & 381 & 381 & 381 & 381 & 381 & 372 & 381 & 381 \\
\hline R2 within & 0.397 & 0.483 & 0.700 & 0.394 & 0.395 & 0.400 & 0.402 & 0.495 & 0.705 & 0.420 & 0.400 & 0.406 \\
\hline R2 between & 0.292 & 0.021 & 0.273 & 0.473 & 0.285 & 0.287 & 0.288 & 0.050 & 0.260 & 0.464 & 0.281 & 0.282 \\
\hline Number of banks & 84 & 84 & 84 & 83 & 84 & 84 & 84 & 84 & 84 & 83 & 84 & 84 \\
\hline Fixed effects & YES & YES & YES & YES & YES & YES & YES & YES & YES & YES & YES & YES \\
\hline Time dummies & YES & YES & YES & YES & YES & YES & YES & YES & YES & YES & YES & YES \\
\hline $\begin{array}{l}\text { Note: Dependent va } \\
\text { Non-operating item } \\
\text { SCORE-3y), Sum o } \\
\text { alternatively for Bar } \\
\text { total assets are less } \\
\text { TL_TA is the ratio } \\
\text { over total assets; GI } \\
* \text { *Sifion }\end{array}$ & bles: Retı & $\begin{array}{l}\text { on assets } \\
\text { average a }\end{array}$ & ided by & $\begin{array}{l}\text { hree-ye } \\
\text { DITAA), }\end{array}$ & $\begin{array}{l}\text { are in brack } \\
\text { vth rate of tot }\end{array}$ & $\begin{array}{l}\text { of Ban } \\
\text { ols incl } \\
\text { NII is }\end{array}$ & $\begin{array}{l}\text { artners } \\
\text { a is th } \\
\text { of non }\end{array}$ & ast ince & $\begin{array}{l}\text { s }(Z \\
\text { SM } \\
\text { asse }\end{array}$ & $\begin{array}{l}\text { a dum } \\
\text { is the } \\
\text { rating i }\end{array}$ & $\begin{array}{l}\text { loan loss pro } \\
\text { OBS is the ra }\end{array}$ & $\begin{array}{l}\text { S ratio (NIEAA), } \\
\text { rn on assets (Z- } \\
\text { le money, stands } \\
\text { lue } 1 \text { if the bank } \\
\text { over total assets; } \\
\text { ff-balance sheet } \\
\text { at the } 5 \% \text { level, }\end{array}$ \\
\hline
\end{tabular}


Table A.2. Mobile money and bank performance in the East African Community: Using the lag (1 year).

\begin{tabular}{|c|c|c|c|c|c|c|c|c|c|}
\hline \multirow{3}{*}{ Panel A } & \multicolumn{4}{|c|}{ ALL BANKS } & \multirow{3}{*}{ Panel B } & \multirow{2}{*}{\multicolumn{4}{|c|}{$\begin{array}{l}\text { COMMERCIAL BANKS } \\
\text { Number of years of Bank-MNO partnership since inception }\end{array}$}} \\
\hline & \multicolumn{4}{|c|}{ Number of years of Bank-MNO partnership since inception } & & & & & \\
\hline & $\begin{array}{l}\text { ROA } \\
(5)\end{array}$ & $\begin{array}{l}\text { ROE } \\
\text { (6) }\end{array}$ & $\begin{array}{l}\text { COST/INCOME } \\
\text { (7) }\end{array}$ & $\begin{array}{l}\text { Z-SCORE } \\
(8)\end{array}$ & & $\begin{array}{l}\text { ROA } \\
(5)\end{array}$ & $\begin{array}{l}\text { ROE } \\
(6)\end{array}$ & $\begin{array}{l}\text { COST/INCOME } \\
(7)\end{array}$ & $\begin{array}{l}\text { Z-SCORE } \\
(8)\end{array}$ \\
\hline MM (t-1) & $\begin{array}{l}0.313^{* * *} \\
(0.108)\end{array}$ & $\begin{array}{l}1.697 * * \\
(0.848)\end{array}$ & $\begin{array}{l}-6.137 * * * \\
(1.638)\end{array}$ & $\begin{array}{l}0.959 * * * \\
(0.340)\end{array}$ & & $\begin{array}{l}0.256^{* *} \\
(0.118)\end{array}$ & $\begin{array}{l}1.416 \\
(0.899)\end{array}$ & $\begin{array}{l}-6.255^{* * *} \\
(1.836)\end{array}$ & $\begin{array}{l}1.190^{* * * *} \\
(0.382)\end{array}$ \\
\hline Controls included & YES & YES & YES & YES & & YES & YES & YES & YES \\
\hline Observations & 466 & 466 & 466 & 466 & & 381 & 381 & 381 & 381 \\
\hline R2 within & 0.319 & 0.263 & 0.390 & 0.098 & & 0.373 & 0.322 & 0.413 & 0.110 \\
\hline R2 between & 0.255 & 0.308 & 0.255 & 0.033 & & 0.294 & 0.353 & 0.263 & 0.013 \\
\hline Number of banks & 103 & 103 & 103 & 103 & & 84 & 84 & 84 & 84 \\
\hline Fixed effects & YES & YES & YES & YES & & YES & YES & YES & YES \\
\hline Time dummies & YES & YES & YES & YES & & YES & YES & YES & YES \\
\hline \multirow{3}{*}{ Panel A } & \multicolumn{4}{|c|}{ ALL BANKS } & \multirow{3}{*}{ Panel B } & \multicolumn{4}{|c|}{ COMMERCIAL BANKS } \\
\hline & \multicolumn{4}{|c|}{ Number of years of Bank-MNO partnership since inception } & & \multicolumn{4}{|c|}{ Number of years of Bank-MNO partnership since inception } \\
\hline & $\begin{array}{l}\text { ROA } \\
\text { (1) }\end{array}$ & $\begin{array}{l}\text { ROE } \\
(2)\end{array}$ & $\begin{array}{l}\text { COST/INCOME } \\
\text { (3) }\end{array}$ & $\begin{array}{l}\text { Z-SCORE } \\
(4)\end{array}$ & & $\begin{array}{l}\text { ROA } \\
(5)\end{array}$ & $\begin{array}{l}\text { ROE } \\
(6)\end{array}$ & $\begin{array}{l}\text { COST/INCOME } \\
(7)\end{array}$ & $\begin{array}{l}\text { Z-SCORE } \\
(8)\end{array}$ \\
\hline MM (t-1) & $\begin{array}{l}0.255^{* *} \\
(0.109)\end{array}$ & $\begin{array}{l}1.274 \\
(0.853)\end{array}$ & $\begin{array}{l}-4.896^{* * * *} \\
(1.623)\end{array}$ & $\begin{array}{l}0.998 * * * \\
(0.346)\end{array}$ & & $\begin{array}{l}-0.257 \\
(0.628)\end{array}$ & $\begin{array}{l}-2.115 \\
(4.820)\end{array}$ & $\begin{array}{l}-1.509 \\
(9.970)\end{array}$ & $\begin{array}{l}3.078 \\
(2.081)\end{array}$ \\
\hline SMALL & $\begin{array}{l}0.749 * * \\
(0.347)\end{array}$ & $\begin{array}{l}5.004^{*} \\
(2.729)\end{array}$ & $\begin{array}{l}-4.372 \\
(5.190)\end{array}$ & $\begin{array}{l}0.479 \\
(1.107)\end{array}$ & & $\begin{array}{l}0.892 * * \\
(0.378)\end{array}$ & $\begin{array}{l}6.168^{* *} \\
(2.903)\end{array}$ & $\begin{array}{l}-14.166^{* *} \\
(6.004)\end{array}$ & $\begin{array}{l}0.917 \\
(1.253)\end{array}$ \\
\hline MM (t-1) x SMALL & $\begin{array}{l}0.454^{*} \\
(0.266)\end{array}$ & $\begin{array}{l}3.599 * \\
(2.093)\end{array}$ & $\begin{array}{l}-15.964 * * * \\
(3.980)\end{array}$ & $\begin{array}{l}-0.815 \\
(0.849)\end{array}$ & & $\begin{array}{l}1.068 \\
(0.796)\end{array}$ & $\begin{array}{l}3.240 \\
(6.105)\end{array}$ & $\begin{array}{l}-7.866 \\
(12.628)\end{array}$ & $\begin{array}{l}-3.914 \\
(2.636)\end{array}$ \\
\hline Total effect & $\begin{array}{l}1.203^{* * * *} \\
(0.379)\end{array}$ & $\begin{array}{l}8.603 * * * \\
(2.983)\end{array}$ & $\begin{array}{l}-20.336 \text { **** } \\
(5.672)\end{array}$ & $\begin{array}{l}-0.336 \\
(1.210)\end{array}$ & & $\begin{array}{l}1.960 * * * \\
(0.788)\end{array}$ & $\begin{array}{l}9.408 \\
(6.051)\end{array}$ & $\begin{array}{l}-22.032^{*} \\
(12.515)\end{array}$ & $\begin{array}{l}-2.997 \\
(2.613)\end{array}$ \\
\hline Controls included & YES & YES & YES & YES & & YES & YES & YES & YES \\
\hline Observations & 466 & 466 & 466 & 466 & & 381 & 381 & 381 & 381 \\
\hline R2 within & 0.339 & 0.280 & 0.423 & 0.100 & & 0.384 & 0.329 & 0.405 & 0.091 \\
\hline R2 between & 0.247 & 0.298 & 0.251 & 0.030 & & 0.297 & 0.356 & 0.273 & 0.015 \\
\hline Number of banks & 103 & 103 & 103 & 103 & & 84 & 84 & 84 & 84 \\
\hline Fixed effects & YES & YES & YES & YES & & YES & YES & YES & YES \\
\hline Time dummies & YES & YES & YES & YES & & YES & YES & YES & YES \\
\hline
\end{tabular}

Note: Dependent variables: return on assets (ROA), return on equity (ROE), Cost to income ratio (COST/INCOME), and the sum of return on assets and equity ratio divided by the standard deviation of return on assets (Z-SCORE). MM (t-1), is the lag 1 year of mobile money, that stands for the number of years of Bank-MNO partnership since inception. SMALL is a dummy variable that takes the value 1 if the bank total assets are less than the median, and 0 otherwise. Robust standard errors are in brackets Controls included: SIZE is the logarithm of total asset; LLP is the ratio of loan loss provision over total assets; TL_TA is the ratio of total loans to total assets; ASSETS Growth is the growth rate of total assets; NII is the share of non-interest income over total operating income; OBS is the ratio of offbalance sheet over total assets; GDP Growth is the annual growth rate of gross domestic product; INFL is the annual growth rate of consumer price index. ***Significant at the $1 \%$ level, **Significant at the $5 \%$ level, *Significant at the $10 \%$ level. 
Table A.3A. Mobile money and bank performance in the East African Community: Using number of mobile money account and value of mobile money transactions.

\begin{tabular}{|c|c|c|c|c|c|c|c|c|}
\hline \multirow{3}{*}{ Panel A } & \multicolumn{8}{|c|}{ ALL BANKS } \\
\hline & \multicolumn{4}{|c|}{ Number of mobile money account (MMU) } & \multicolumn{4}{|c|}{ Value of mobile money transactions (MMT) } \\
\hline & $\begin{array}{l}\text { ROA } \\
\text { (1) }\end{array}$ & $\begin{array}{l}\text { ROE } \\
\text { (2) }\end{array}$ & $\begin{array}{l}\text { COST/INCOME } \\
\text { (3) }\end{array}$ & $\begin{array}{l}\text { Z-SCORE } \\
\text { (4) }\end{array}$ & $\begin{array}{l}\text { ROA } \\
(5)\end{array}$ & $\begin{array}{l}\text { ROE } \\
(6)\end{array}$ & $\begin{array}{l}\text { COST/INCOME } \\
\text { (7) }\end{array}$ & $\begin{array}{l}\text { Z-SCORE } \\
\text { (8) }\end{array}$ \\
\hline \multirow[t]{2}{*}{ MM } & $0.721 * * *$ & $6.668 * * *$ & $-14.482 * * *$ & -0.310 & $0.372 * * *$ & $3.219 * * *$ & $-6.867 * * *$ & -0.183 \\
\hline & $(0.217)$ & $(1.683)$ & $(3.283)$ & $(0.694)$ & $(0.078)$ & $(0.597)$ & $(1.171)$ & $(0.252)$ \\
\hline Controls included & YES & YES & YES & YES & YES & YES & YES & YES \\
\hline Observations & 457 & 457 & 457 & 457 & 450 & 450 & 450 & 450 \\
\hline R2 within & 0.325 & 0.286 & 0.401 & 0.082 & 0.346 & 0.321 & 0.420 & 0.082 \\
\hline $\mathbf{R} 2$ between & 0.256 & 0.312 & 0.263 & 0.018 & 0.272 & 0.327 & 0.271 & 0.018 \\
\hline Number of banks & 101 & 101 & 101 & 101 & 101 & 101 & 101 & 101 \\
\hline Fixed effects & YES & YES & YES & YES & YES & YES & YES & YES \\
\hline \multirow[t]{2}{*}{ Time dummies } & YES & YES & YES & YES & YES & YES & YES & YES \\
\hline & \multicolumn{8}{|c|}{ COMMERCIAL BANKS } \\
\hline \multirow[t]{3}{*}{ Panel B } & \multicolumn{4}{|c|}{ Number of mobile money account (MMU) } & \multicolumn{4}{|c|}{ Value of mobile money transactions (MMT) } \\
\hline & ROA & ROE & COST/INCOME & Z-SCORE & ROA & ROE & COST/INCOME & Z-SCORE \\
\hline & (1) & $(2)$ & (3) & (4) & $(5)$ & (6) & $(7)$ & (8) \\
\hline \multirow[t]{2}{*}{ MM } & $0.687 * * *$ & $5.889 * * *$ & $-14.416^{* * *}$ & -0.197 & $0.431 * * *$ & $3.710 * * *$ & $-7.859 * * *$ & -0.144 \\
\hline & $(0.236)$ & $(1.796)$ & $(3.711)$ & $(0.788)$ & $(0.085)$ & $(0.636)$ & $(1.320)$ & $(0.288)$ \\
\hline Controls included & YES & YES & YES & YES & YES & YES & YES & YES \\
\hline Observations & 372 & 372 & 372 & 372 & 366 & 366 & 366 & 366 \\
\hline R2 within & 0.384 & 0.343 & 0.422 & 0.086 & 0.423 & 0.395 & 0.465 & 0.085 \\
\hline $\mathbf{R} 2$ between & 0.297 & 0.361 & 0.277 & 0.013 & 0.322 & 0.387 & 0.290 & 0.011 \\
\hline Number of banks & 82 & 82 & 82 & 82 & 82 & 82 & 82 & 82 \\
\hline Fixed effects & YES & YES & YES & YES & YES & YES & YES & YES \\
\hline Time dummies & YES & YES & YES & YES & YES & YES & YES & YES \\
\hline
\end{tabular}


Table A.3B. Mobile money and bank performance in the East African Community (Small vs. Large banks): Using number of mobile money account and value of mobile money transactions.

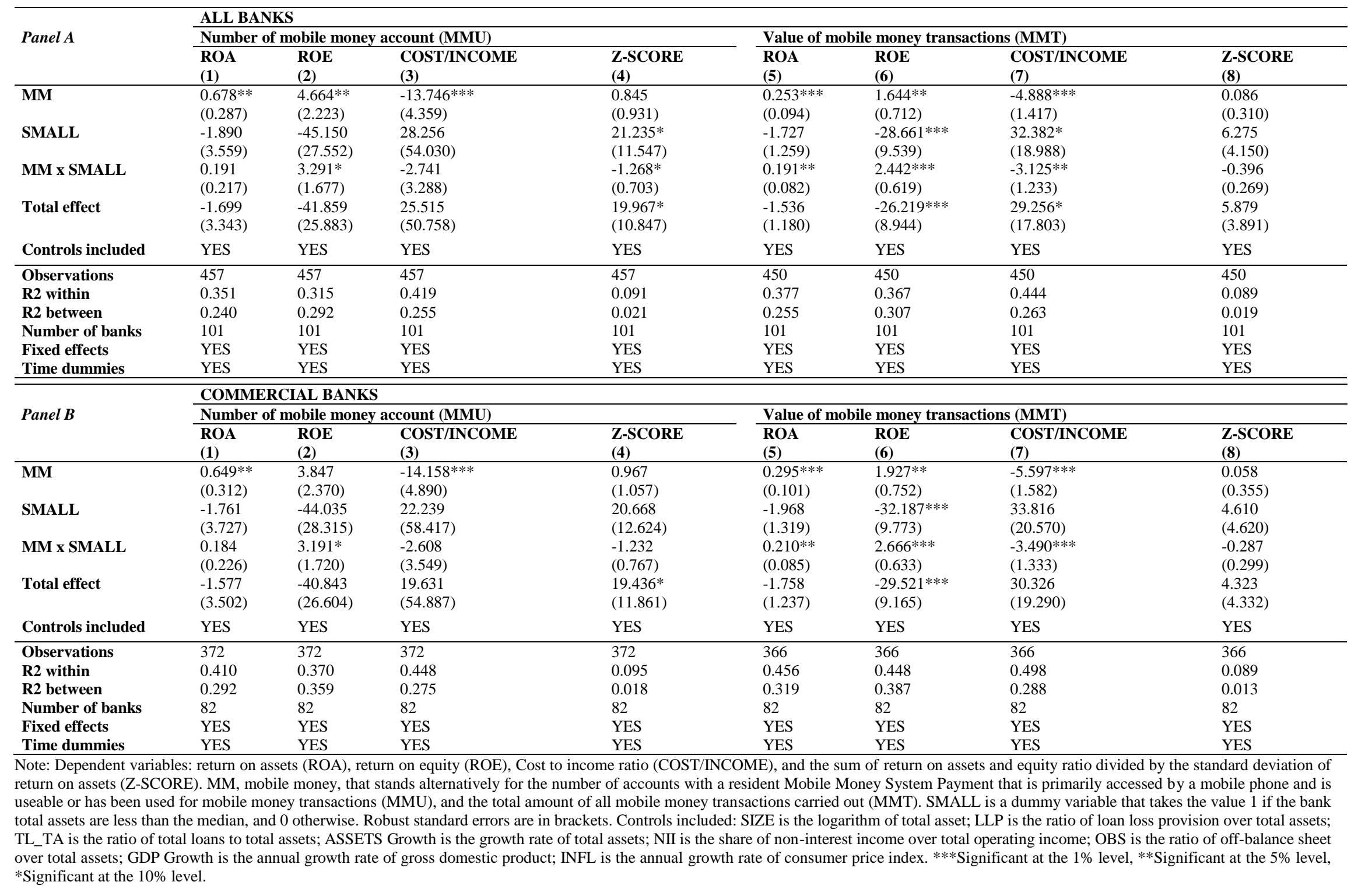


Table A.4. Mobile money and bank performance in the East African Community: All financial institutions.

\begin{tabular}{|c|c|c|c|c|c|c|c|c|}
\hline \multirow{3}{*}{ Panel A } & \multicolumn{8}{|c|}{ ALL FINANCIAL INSTITUTIONS } \\
\hline & \multicolumn{4}{|c|}{ Bank-MNO partnership (1 if yes, 0 otherwise) } & \multicolumn{4}{|c|}{ Number of years of Bank-MNO partnership since inception } \\
\hline & $\begin{array}{l}\text { ROA } \\
\text { (1) }\end{array}$ & $\begin{array}{l}\text { ROE } \\
(2)\end{array}$ & $\begin{array}{l}\text { COST/INCOME } \\
\text { (3) }\end{array}$ & $\begin{array}{l}\text { Z-SCORE } \\
\text { (4) }\end{array}$ & $\begin{array}{l}\text { ROA } \\
(5)\end{array}$ & $\begin{array}{l}\text { ROE } \\
\text { (6) }\end{array}$ & $\begin{array}{l}\text { COST/INCOME } \\
\text { (7) }\end{array}$ & $\begin{array}{l}\text { Z-SCORE } \\
(8)\end{array}$ \\
\hline MM & $\begin{array}{l}1.306^{* * *} \\
(0.590)\end{array}$ & $\begin{array}{l}39.352 * \\
(22.870)\end{array}$ & $\begin{array}{l}-9.821 \\
(6.218)\end{array}$ & $\begin{array}{l}0.554 * * \\
(0.228)\end{array}$ & $\begin{array}{l}0.444 * * * \\
(0.149)\end{array}$ & $\begin{array}{l}7.836 \\
(5.814)\end{array}$ & $\begin{array}{l}-6.400 * * * \\
(1.545)\end{array}$ & $\begin{array}{l}1.012 * * * \\
(0.322)\end{array}$ \\
\hline Controls included & YES & YES & YES & YES & YES & YES & YES & YES \\
\hline Observations & 490 & 490 & 489 & 490 & 490 & 490 & 489 & 490 \\
\hline R2 within & 0.548 & 0.624 & 0.368 & 0.202 & 0.553 & 0.623 & 0.392 & 0.107 \\
\hline R2 between & 0.456 & 0.516 & 0.262 & 0.126 & 0.445 & 0.518 & 0.257 & 0.025 \\
\hline Number of banks & 110 & 110 & 110 & 110 & 110 & 110 & 110 & 110 \\
\hline Fixed effects & YES & YES & YES & YES & YES & YES & YES & YES \\
\hline \multirow[t]{2}{*}{ Time dummies } & YES & YES & YES & YES & YES & YES & YES & YES \\
\hline & \multicolumn{8}{|c|}{ ALL FINANCIAL INSTITUTIONS } \\
\hline \multirow{2}{*}{ Panel B } & \multicolumn{4}{|c|}{ Bank-MNO partnership (1 if yes, 0 otherwise) } & \multicolumn{4}{|c|}{ Number of years of Bank-MNO partnership since inception } \\
\hline & $\begin{array}{l}\text { ROA } \\
\text { (1) }\end{array}$ & $\begin{array}{l}\text { ROE } \\
(2)\end{array}$ & $\begin{array}{l}\text { COST/INCOME } \\
\text { (3) }\end{array}$ & $\begin{array}{l}\text { Z-SCORE } \\
(4)\end{array}$ & $\begin{array}{l}\text { ROA } \\
(\mathbf{5})\end{array}$ & $\begin{array}{l}\text { ROE } \\
(6)\end{array}$ & $\begin{array}{l}\text { COST/INCOME } \\
\text { (7) }\end{array}$ & $\begin{array}{l}\text { Z-SCORE } \\
(8)\end{array}$ \\
\hline MM & $\begin{array}{l}0.610 \\
(0.659)\end{array}$ & $\begin{array}{l}33.757 \\
(25.849)\end{array}$ & $\begin{array}{l}-2.169 \\
(6.959)\end{array}$ & $\begin{array}{l}0.404 \\
(0.257)\end{array}$ & $\begin{array}{l}0.377 * * \\
(0.151)\end{array}$ & $\begin{array}{l}7.783 \\
(5.926)\end{array}$ & $\begin{array}{l}-5.364 * * * \\
(1.521)\end{array}$ & $\begin{array}{l}1.020 \text { **** } \\
(0.327)\end{array}$ \\
\hline SMALL & $\begin{array}{l}0.882 * * \\
(0.444)\end{array}$ & $\begin{array}{l}8.858 \\
(17.433)\end{array}$ & $\begin{array}{l}-4.427 \\
(4.683)\end{array}$ & $\begin{array}{l}0.067 \\
(0.173)\end{array}$ & $\begin{array}{l}0.868^{*} \\
(0.448)\end{array}$ & $\begin{array}{l}11.948 \\
(17.609)\end{array}$ & $\begin{array}{l}0.767 \\
(4.514)\end{array}$ & $\begin{array}{l}0.992 \\
(0.973)\end{array}$ \\
\hline MM x SMALL & $\begin{array}{l}1.848 * * \\
(0.936)\end{array}$ & $\begin{array}{l}14.372 \\
(36.741)\end{array}$ & $\begin{array}{l}-21.806^{* * *} \\
(9.869)\end{array}$ & $\begin{array}{l}0.433 \\
(0.366)\end{array}$ & $\begin{array}{l}0.359 \\
(0.369)\end{array}$ & $\begin{array}{l}-10.666 \\
(14.498)\end{array}$ & $\begin{array}{l}-18.971 * * * \\
(3.722)\end{array}$ & $\begin{array}{l}-1.111 \\
(0.801)\end{array}$ \\
\hline Total effect & $\begin{array}{l}2.730 * * * \\
(0.938)\end{array}$ & $\begin{array}{l}23.230 \\
(36.812)\end{array}$ & $\begin{array}{l}-26.233 * * * \\
(9.888)\end{array}$ & $\begin{array}{l}0.501 \\
(0.366)\end{array}$ & $\begin{array}{l}1.227 * * * \\
(0.512)\end{array}$ & $\begin{array}{l}1.282 \\
(20.102)\end{array}$ & $\begin{array}{l}-18.204^{* * * *} \\
(5.153)\end{array}$ & $\begin{array}{l}0.232 \\
(0.195)\end{array}$ \\
\hline Controls included & YES & YES & YES & YES & YES & YES & YES & YES \\
\hline Observations & 490 & 490 & 489 & 490 & 490 & 490 & 489 & 490 \\
\hline R2 within & 0.561 & 0.625 & 0.380 & 0.206 & 0.560 & 0.624 & 0.434 & 0.112 \\
\hline R2 between & 0.459 & 0.522 & 0.257 & 0.110 & 0.449 & 0.517 & 0.246 & 0.015 \\
\hline Number of banks & 110 & 110 & 110 & 110 & 110 & 110 & 110 & 110 \\
\hline Fixed effects & YES & YES & YES & YES & YES & YES & YES & YES \\
\hline Time dummies & YES & YES & YES & YES & YES & YES & YES & YES \\
\hline
\end{tabular}

Note: Dependent variables: Return on assets (ROA), Return on equity (ROE), Cost to income ratio (COST/INCOME), and the sum of return on assets and equity ratio divided by the standard deviation of return on assets (Z-SCORE). MM, involvement in mobile money, stands alternatively for Bank-MNO partnership that equals 1 if yes, 0 otherwise, and the number of years of Bank-MNO partnership since inception. SMALL is a dummy variable that takes the value 1 if the bank total assets are less than the median, and 0 otherwise. Robust standard errors are in brackets. Controls included: SIZE is the logarithm of total asset; LLP is the ratio of loan loss provision over total assets; TL_TA is the ratio of total loans to total assets; ASSETS Growth is the growth rate of total assets; NII is the share of noninterest income over total operating income; OBS is the ratio of off-balance sheet over total assets; GDP Growth is the annual growth rate of gross domestic product; INFL is the annual growth rate of consumer price index. $* * *$ Significant at the $1 \%$ level, $* *$ Significant at the $5 \%$ level, *Significant at the $10 \%$ level. 
Table A.5. Descriptive statistics.

\begin{tabular}{|c|c|c|c|c|c|c|c|c|c|c|}
\hline & Obs. & Mean & Std. Dev. & Min & Max & Obs. & Mean & Std. Dev. & Min & Max \\
\hline & \multirow{2}{*}{\multicolumn{5}{|c|}{ All financial institutions }} & \multirow{2}{*}{\multicolumn{5}{|c|}{ Commercial banks }} \\
\hline \multicolumn{7}{|l|}{ Dependent variable } & & & & \\
\hline ROA & 715 & 0.940 & 6.401 & -81.973 & 26.632 & 522 & 1.100 & 4.261 & -32.545 & 26.632 \\
\hline ROE & 715 & -4.456 & 202.602 & -4225 & 76.545 & 522 & 7.033 & 33.987 & -567.795 & 45.129 \\
\hline Z-SCORE & 712 & 30.069 & 44.031 & -2.241 & 540.320 & 521 & 26.736 & 28.684 & -1.450 & 348.339 \\
\hline COST/INCOME & 685 & 78.325 & 64.742 & 5.75 & 876.839 & 499 & 77.269 & 67.445 & 5.750 & 876.839 \\
\hline RAROA & 715 & 0.156 & 0.916 & -10.898 & 4.971 & 522 & 0.247 & 0.922 & -7.941 & 6.097 \\
\hline NIM & 691 & 7.133 & 5.381 & -0.437 & 41.381 & 501 & 5.921 & 3.087 & -0.437 & 41.381 \\
\hline NIEAA & 694 & 10.263 & 8.835 & 0.220 & 68.608 & 504 & 8.373 & 6.541 & 0.220 & 68.608 \\
\hline NOITAA & 669 & -0.625 & 1.645 & -8.577 & 18.868 & 488 & -0.595 & 1.238 & -5.215 & 13.849 \\
\hline Z-SCORE (3y) & 715 & 0.184 & 0.918 & -10.896 & 5.077 & 522 & 0.284 & 0.921 & -7.862 & 6.323 \\
\hline Z-SCORE (5y) & 715 & 0.166 & 0.890 & -10.896 & 4.274 & 522 & 0.280 & 0.904 & -7.093 & 6.609 \\
\hline \multicolumn{11}{|l|}{ Variable of interest (Mobile money - MM) } \\
\hline Involvement in MM & 1,19 & 0.183 & 0.387 & 0 & 1 & 847 & 0.191 & 0.394 & 0 & 1 \\
\hline Number of years of Bank-MNO partnership since inception & 1,19 & 0.697 & 1.748 & 0 & 9 & 847 & 0.769 & 1.866 & 0 & 9 \\
\hline Number of mobile money account & 1,124 & 16.386 & 1.118 & 12.206 & 17.802 & 790 & 16.415 & 1.107 & 12.206 & 17.802 \\
\hline Value of mobile money transactions (\% GDP) & 1,114 & 14.924 & 2.713 & 6.142 & 17.126 & 782 & 14.996 & 2.605 & 6.142 & 17.126 \\
\hline \multicolumn{11}{|l|}{$\begin{array}{l}\text { Control variables } \\
\end{array}$} \\
\hline$\overline{\text { SIZE }}$ & 718 & 12.120 & 1.380 & 5.834 & 15.335 & 523 & 12.348 & 1.246 & 8.129 & 15.335 \\
\hline LLP & 655 & 0.971 & 1.702 & -3.114 & 17.711 & 477 & 0.749 & 1.095 & -3.114 & 8.845 \\
\hline TL_TA & 699 & 52.746 & 14.454 & 0.887 & 98.144 & 519 & 50.220 & 12.599 & 0.887 & 87.414 \\
\hline ASSET Growth & 578 & 18.420 & 34.791 & -85.339 & 477.546 & 423 & 17.244 & 32.318 & -74.689 & 477.546 \\
\hline NII & 712 & 0.367 & 0.229 & -1.143 & 2.834 & 519 & 0.379 & 0.189 & -0.480 & 1.111 \\
\hline OBS & 618 & 0.154 & 0.145 & 0.000 & 1.453 & 485 & 0.166 & 0.136 & 0.000 & 0.914 \\
\hline GDP Growth & 1,19 & 5.836 & 1.736 & -3.905 & 9.439 & 847 & 5.835 & 1.767 & -3.905 & 9.439 \\
\hline INFL & 1,19 & 7.977 & 4.613 & -5.315 & 21.521 & 847 & 8.022 & 4.412 & -5.315 & 21.521 \\
\hline \multirow[t]{3}{*}{ SMALL } & 1,19 & 0.305 & 0.461 & 0 & 1 & 847 & 0.295 & 0.456 & 0 & 1 \\
\hline & Obs. & Mean & Std. Dev. & Min & Max & Obs. & Mean & Std. Dev. & Min & Max \\
\hline & \multicolumn{5}{|c|}{ Small banks } & \multicolumn{5}{|c|}{ Large banks } \\
\hline \multicolumn{11}{|l|}{ Dependent variable } \\
\hline ROA & 329 & 0.059 & 5.494 & -32.545 & 21.619 & 321 & 2.467 & 2.209 & -3.392 & 26.632 \\
\hline ROE & 329 & -1.168 & 41.488 & -567.795 & 76.545 & 321 & 15.685 & 11.985 & -52.811 & 45.129 \\
\hline Z-SCORE & 312 & 96.334 & 86.616 & 5.750 & 876.839 & 311 & 57.540 & 22.399 & 8.179 & 254.269 \\
\hline COST/INCOME & 327 & 29.199 & 50.092 & -1.450 & 386.599 & 321 & 30.547 & 26.471 & 1.244 & 277.527 \\
\hline RAROA & 329 & .021 & 1.189 & -7.941 & 5.243 & 321 & 0.549 & 0.501 & -0.811 & 6.097 \\
\hline NIM & 315 & 6.124 & 4.534 & -0.437 & 35.926 & 311 & 6.263 & 2.837 & 0.139 & 41.381 \\
\hline NIEAA & 318 & 11.400 & 8.641 & 0.220 & 68.608 & 311 & 6.384 & 2.816 & 1.049 & 17.124 \\
\hline NOITAA & 303 & -0.330 & 1.883 & -8.577 & 13.849 & 306 & -0.957 & 0.723 & -5.215 & 1.621 \\
\hline Z-SCORE (3y) & 329 & 0.067 & 1.190 & -7.862 & 5.355 & 321 & 0.584 & 0.514 & -0.795 & 6.323 \\
\hline Z-SCORE (5y) & 329 & 0.065 & 1.161 & -7.093 & 5.219 & 321 & 0.576 & 0.517 & -0.719 & 6.609 \\
\hline \multicolumn{11}{|l|}{ Variable of interest (Mobile money - MM) } \\
\hline Involvement in MM & 330 & 0.103 & 0.304 & 0 & 1 & 741 & 0.223 & 0.416 & 0 & 1 \\
\hline Number of years of Bank-MNO partnership since inception & 330 & 0.282 & 0.987 & 0 & 7 & 741 & 0.924 & 2.025 & 0 & 9 \\
\hline Number of mobile money account & 307 & 16.252 & 1.170 & 12.206 & 17.802 & 699 & 16.472 & 1.071 & 12.206 & 17.802 \\
\hline Value of mobile money transactions (\% GDP) & 302 & 14.632 & 2.630 & 6.142 & 17.126 & 695 & 15.107 & 2.669 & 6.142 & 17.126 \\
\hline \multicolumn{11}{|l|}{ Control variables } \\
\hline$\overline{\text { SIZE }}$ & 330 & 11.274 & 0.802 & 8.129 & 12.144 & 322 & 13.268 & 0.822 & 12.151 & 15.335 \\
\hline LLP & 291 & 1.148 & 2.189 & -3.114 & 17.711 & 300 & 0.719 & 0.900 & -0.666 & 8.845 \\
\hline TL_TA & 318 & 50.840 & 15.338 & 0.887 & 98.144 & 320 & 52.574 & 12.749 & 5.241 & 87.414 \\
\hline ASSET Growth & 245 & 22.777 & 43.127 & -85.339 & 477.546 & 283 & 12.718 & 16.399 & -74.689 & 81.010 \\
\hline NII & 326 & 0.405 & 0.229 & -0.460 & 1.111 & 321 & 0.345 & 0.170 & -0.480 & 1 \\
\hline OBS & 283 & 0.136 & 0.134 & 0.000 & 0.914 & 307 & 0.181 & 0.153 & 0.000 & 1.453 \\
\hline GDP Growth & 330 & 5.862 & 1.589 & 3.307 & 9.439 & 741 & 5.814 & 1.818 & -3.905 & 9.439 \\
\hline INFL & 330 & 8.367 & 4.606 & -5.315 & 21.521 & 741 & 7.850 & 4.485 & -5.315 & 21.521 \\
\hline
\end{tabular}




\section{Appendix A.6. Overview of the banking system in the East African Community}

The relative depth of the banking system to finance domestic investments is illustrated by the level of domestic credit to the private sector by banks (Table A.6B). Over the last seven years, the annual growth in domestic credit to the private sector during the period 2009-15 averaged 30\% in Kenya, 17\% in Rwanda, 16\% in Burundi and 13\% in Uganda and Tanzania. During the last two decades, economies across the region have successfully unravelled state-controlled banking systems, restructured loss-making institutions, written off nonperforming loans, and improved governance and financial sector supervision (Lepetit et al., 2015; Sanya and Gaertner, 2012). Over the period of 2009-2015, the average ratio of the non-performing loans over total gross loans is $6 \%$. Trends are quite similar across countries where the averages are in Uganda 4\%, Tanzania and Rwanda 6\% while Burundi exhibits a relative higher level of non-performing loans ratio averaging $9 \%$.

The financial system in the EAC is dominated by commercial banks and reveals disparities among the countries (see Table A.6A). As regards to the structure of the banking market in the $\mathrm{EAC}$, it is characterized by lower levels of competition between banks. The bank lending-deposit spreads are relatively high with Uganda and Kenya banking systems displaying the highest levels (11\% and $8 \%$ respectively) and this is also reflected in their comparatively high return on assets and return on equity that stand respectively at $3 \%$ and $18 \%$ on average over the period. The banking performance indicators also reveal that in the region the banking sector of Rwanda is relatively less profitable. In the recent years, the lightning innovation of mobile money has moved to the core of banking system. Stijns and Revoltella (2016) argue that whereas mobile money has largely been characterized as a technological innovation, it has actually been more of an enabler supporting an innovative banking model. The mobile money system is promoting financial inclusion by reducing transactional costs and contributing to the surge of new financial intermediation services. By reducing the need for cash for transactions, the increasing adoption of mobile money may allow economic agents to capture financial market signals therefore improving the environment for monetary policy and financial development. In the EAC region, more than anywhere else, mobile money has helped integrate large sections of the population into the financial system. Tanzania is the country that records the highest number of financial institutions (16) involved in mobile money system, followed by Kenya with 14 and Uganda with 11 financial institutions, Rwanda with 5 and Burundi with only 2 financial institution as of 2015. 
Table A.6A. Sample of financial institutions in the EAC over 2009-2015.

\begin{tabular}{lll}
\hline Financial institutions specialization & Frequency & Percent \\
\hline Bank holdings \& Holding companies & 2 & 1.18 \\
Commercial banks & 121 & 71.18 \\
Cooperative banks & 2 & 1.18 \\
Finance companies & 8 & 4.71 \\
Investment \& Trust corporations & 2 & 1.18 \\
Investment banks & 5 & 2.94 \\
Islamic banks & 3 & 1.76 \\
Micro-financing institutions & 17 & 10.00 \\
Multi-lateral governmental banks & 1 & 0.59 \\
Private banking / Asset management comp & 1 & 0.59 \\
Real Estate \& Mortgage banks & 2 & 1.18 \\
Savings banks & 2 & 1.18 \\
Specialized governmental credit institution & 4 & 2.35 \\
\hline So & & \\
\hline
\end{tabular}

Source: Authors' analysis of the data retrieved from Bankscope.

Table A.6B. EAC financial system indicators over the period $2009-2015$.

\begin{tabular}{|c|c|c|c|c|c|c|c|c|}
\hline & 2009 & 2010 & 2011 & 2012 & 2013 & 2014 & 2015 & Average \\
\hline \multicolumn{9}{|c|}{ Bank nonperforming loans to total gross loans (\%) } \\
\hline Burundi & n.a. & 9.32 & 7.39 & 8.17 & 9.89 & 10.82 & 17.92 & 9.07 \\
\hline Kenya & 8.00 & 6.29 & 4.43 & 4.59 & 5.05 & 5.46 & 5.99 & 5.69 \\
\hline Rwanda & 8.58 & 7.33 & 5.59 & 5.11 & 5.93 & 5.22 & 5.91 & 6.24 \\
\hline Tanzania & n.a. & 7.84 & 5.40 & 6.40 & 5.12 & 6.58 & 8.58 & 5.70 \\
\hline Uganda & 3.97 & 1.86 & 2.03 & 4.06 & 5.76 & 4.01 & 5.13 & 3.83 \\
\hline \multicolumn{9}{|c|}{ Domestic credit to private sector by banks (\% of GDP) } \\
\hline$\overline{\text { Burundi }}$ & 14.78 & 16.68 & 18.68 & 17.58 & 16.03 & 15.11 & 13.95 & 16.12 \\
\hline Kenya & 24.93 & 27.13 & 30.42 & 29.48 & 31.63 & 34.07 & 34.62 & 30.33 \\
\hline Rwanda & 11.57 & 12.03 & 15.781 & 18.28 & 18.98 & 20.53 & 20.91 & 16.87 \\
\hline Tanzania & 11.20 & 11.73 & 12.49 & 12.91 & 12.82 & 13.71 & 15.03 & 12.84 \\
\hline Uganda & 9.72 & 13.28 & 15.14 & 13.41 & 13.31 & 13.80 & 14.33 & 13.29 \\
\hline \multicolumn{9}{|c|}{ Bank lending-deposit spread \% } \\
\hline$\overline{\text { Burundi }}$ & n.a. & n.a. & n.a. & n.a. & n.a. & n.a. & n.a. & n.a. \\
\hline Kenya & 6.9 & 8.14 & 8.67 & 8.15 & 9.42 & 9.81 & 8.84 & 8.56 \\
\hline Rwanda & 9.44 & 9.9 & 8.35 & 6.45 & 8.77 & 9.84 & 7.23 & 8.57 \\
\hline Tanzania & 6.21 & 6.41 & 6.02 & 5.95 & 8.18 & 7.98 & 7.06 & 6.83 \\
\hline Uganda & 9.83 & 10.72 & 11.41 & 10.08 & 8.81 & 12.49 & 11.2 & 10.65 \\
\hline \multicolumn{9}{|c|}{ Return on assets \% } \\
\hline$\overline{\text { Burundi }}$ & 2.95 & 2.41 & 3.44 & 1.91 & 1.08 & 0.74 & n.a. & 1.79 \\
\hline Kenya & 2.5 & 3.45 & 3.1 & 2.84 & 3.23 & 3.38 & 2.73 & 3.03 \\
\hline Rwanda & 0.94 & 1.47 & 1.97 & 1.84 & 0.52 & 1.47 & 1.58 & 1.40 \\
\hline Tanzania & 1.95 & 1.49 & 1.37 & 1.56 & 1.69 & 1.82 & 1.64 & 1.65 \\
\hline Uganda & 3,00 & 2.51 & 3.85 & 3.72 & 2.6 & 2.87 & 2.7 & 3.04 \\
\hline \multicolumn{9}{|c|}{ Return on equity \% } \\
\hline Burundi & 22.36 & 21.04 & 24.74 & 13.48 & 9.05 & 5.69 & n.a. & 13.77 \\
\hline Kenya & 16.64 & 21.07 & 21.21 & 17.74 & 19.5 & 18.51 & 15.48 & 18.59 \\
\hline Rwanda & 6,00 & 9.58 & 11.56 & 10.28 & 3.46 & 10.18 & 14.5 & 9.37 \\
\hline Tanzania & 17.14 & 12.2 & 12.78 & 13.7 & 12.4 & 13.29 & 11.6 & 13.30 \\
\hline Uganda & 18.34 & 17.65 & 24.07 & 21.48 & 15.18 & 16.51 & 16.75 & 18.57 \\
\hline \multicolumn{9}{|c|}{ Number of financial institutions involved in mobile money } \\
\hline Burundi & 1 & 1 & 1 & 2 & 2 & 2 & 2 & - \\
\hline Kenya & 9 & 9 & 9 & 9 & 9 & 10 & 14 & - \\
\hline Rwanda & 1 & 2 & 2 & 2 & 5 & 5 & 5 & - \\
\hline Tanzania & 7 & 7 & 7 & 8 & 14 & 16 & 16 & - \\
\hline Uganda & 5 & 6 & 6 & 7 & 10 & 10 & 11 & . \\
\hline
\end{tabular}

Source: World Development Indicators and Global financial Development from World Bank Group (except the number of financial institutions involved in mobile money supply). Data on mobile money are hand collected. 


\section{Table A.7. Correlation matrix.}

\begin{tabular}{|c|c|c|c|c|c|c|c|c|c|c|c|c|c|c|c|c|c|c|c|c|c|c|c|}
\hline & ROA & ROE & COST/INCOME & $\begin{array}{l}\text { Z- } \\
\text { SCORE }\end{array}$ & RAROA & NIM & NIEAA & NOITAA & $\begin{array}{l}\text { Z- } \\
\text { SCORE } \\
(3 y)\end{array}$ & $\begin{array}{l}\text { Z- } \\
\text { SCORE } \\
\text { (5y) } \\
\end{array}$ & $\begin{array}{l}\text { Involvement } \\
\text { in MM }\end{array}$ & $\begin{array}{l}\text { Number of years } \\
\text { of involvement }\end{array}$ & MMU & MMT & SIZE & LLP & TL_TA & $\begin{array}{l}\text { ASSET } \\
\text { Growth }\end{array}$ & NII & OBS & $\begin{array}{l}\text { GDP } \\
\text { Growth }\end{array}$ & INFL & SMALL \\
\hline ROA & 1.000 & & & & & & & & & & & & & & & & & & & & & & \\
\hline ROE & 0.679 & 1.000 & & & & & & & & & & & & & & & & & & & & & \\
\hline COST/INCOME & -0.773 & -0.538 & 1.000 & & & & & & & & & & & & & & & & & & & & \\
\hline Z-SCORE & 0.131 & 0.103 & -0.177 & 1.000 & & & & & & & & & & & & & & & & & & & \\
\hline RAROA & 0.994 & 0.651 & -0.742 & 0.129 & 1.000 & & & & & & & & & & & & & & & & & & \\
\hline NIM & 0.297 & -0.011 & -0.199 & 0.030 & 0.307 & 1.000 & & & & & & & & & & & & & & & & & \\
\hline NIEAA & -0.561 & -0.538 & 0.562 & -0.070 & -0.540 & 0.271 & 1.000 & & & & & & & & & & & & & & & & \\
\hline NOITAA & -0.763 & -0.615 & 0.663 & -0.080 & -0.755 & -0.313 & 0.295 & 1.000 & & & & & & & & & & & & & & & \\
\hline Z-SCORE (3y) & 0.992 & 0.649 & -0.737 & 0.144 & 0.999 & 0.313 & -0.531 & -0.750 & 1.000 & & & & & & & & & & & & & & \\
\hline Z-SCORE (5y) & 0.993 & 0.650 & -0.736 & 0.144 & 0.998 & 0.316 & -0.531 & -0.747 & 0.999 & 1.000 & & & & & & & & & & & & & \\
\hline $\begin{array}{l}\text { Involvement in } \\
\text { MM }\end{array}$ & 0.146 & 0.128 & -0.091 & -0.037 & 0.150 & 0.066 & -0.116 & -0.121 & 0.145 & 0.147 & 1.000 & & & & & & & & & & & & \\
\hline $\begin{array}{l}\text { Number of years } \\
\text { of involvement }\end{array}$ & 0.180 & 0.144 & -0.128 & -0.024 & 0.191 & 0.108 & -0.128 & -0.154 & 0.189 & 0.192 & 0.847 & 1.000 & & & & & & & & & & & \\
\hline MMU & 0.030 & 0.082 & -0.108 & 0.067 & 0.034 & -0.084 & -0.106 & -0.052 & 0.037 & 0.038 & 0.056 & 0.153 & 1.000 & & & & & & & & & & \\
\hline SIZE & 0.327 & 0.357 & -0.385 & -0.033 & 0.319 & -0.034 & -0.519 & -0.219 & 0.308 & 0.310 & 0.468 & 0.496 & 0.163 & 0.179 & 1.000 & & & & & & & & \\
\hline LLP & -0.145 & -0.173 & -0.043 & 0.320 & -0.150 & 0.352 & 0.370 & 0.092 & -0.134 & -0.136 & -0.081 & -0.075 & 0.005 & 0.010 & -0.126 & 1.000 & & & & & & & \\
\hline TL_TA & 0.324 & 0.178 & -0.247 & -0.127 & 0.327 & 0.271 & -0.086 & -0.321 & 0.320 & 0.321 & -0.079 & -0.050 & 0.108 & 0.163 & 0.098 & -0.100 & 1.000 & & & & & & \\
\hline ASSET Growth & -0.295 & -0.209 & 0.399 & -0.043 & -0.291 & -0.174 & 0.328 & 0.353 & -0.290 & -0.293 & -0.137 & -0.172 & -0.085 & -0.051 & -0.212 & -0.119 & -0.108 & 1.000 & & & & & \\
\hline NII & -0.102 & -0.093 & 0.291 & -0.059 & -0.099 & -0.414 & 0.349 & 0.069 & -0.100 & -0.099 & 0.009 & -0.038 & -0.073 & -0.059 & -0.156 & -0.077 & -0.135 & 0.136 & 1.000 & & & & \\
\hline OBS & 0.014 & 0.007 & 0.005 & -0.043 & 0.016 & -0.090 & -0.069 & -0.064 & 0.017 & 0.018 & 0.080 & 0.076 & 0.020 & 0.084 & 0.143 & -0.107 & 0.155 & 0.018 & 0.001 & 1.000 & & & \\
\hline GDP Growth & -0.023 & 0.003 & 0.036 & 0.004 & -0.024 & -0.040 & 0.026 & 0.010 & -0.026 & -0.022 & 0.047 & 0.029 & -0.109 & -0.113 & 0.027 & -0.064 & -0.006 & 0.041 & 0.015 & -0.020 & 1.000 & & \\
\hline INFL & -0.015 & -0.009 & 0.023 & -0.003 & -0.010 & 0.024 & 0.026 & -0.010 & -0.010 & -0.017 & -0.045 & -0.049 & 0.034 & 0.146 & -0.069 & 0.032 & -0.013 & -0.043 & 0.035 & -0.045 & -0.303 & 1.000 & \\
\hline SMALL & -0.276 & -0.265 & 0.293 & -0.017 & -0.277 & -0.018 & 0.363 & 0.216 & -0.271 & -0.273 & -0.142 & -0.165 & -0.092 & -0.082 & -0.776 & 0.128 & -0.061 & 0.156 & 0.147 & -0.153 & 0.013 & 0.053 & 1.000 \\
\hline
\end{tabular}

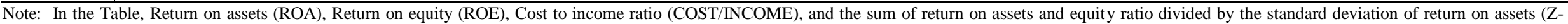

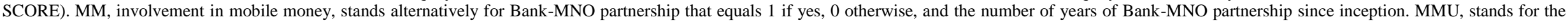

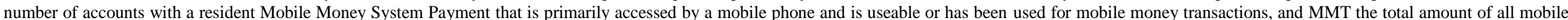

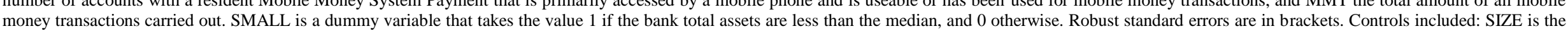

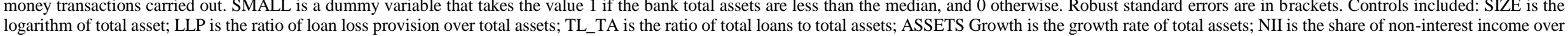
total operating income; OBS is the ratio of off-balance sheet over total assets; GDP Growth is the annual growth rate of gross domestic product; INFL is the annual growth rate of consumer price index. 
Figure A1. Adoption rates of mobile financial services by geographic region (EAC averages, 2017 or the latest for Burundi).

1. Mobile money account (\% age $15+)$

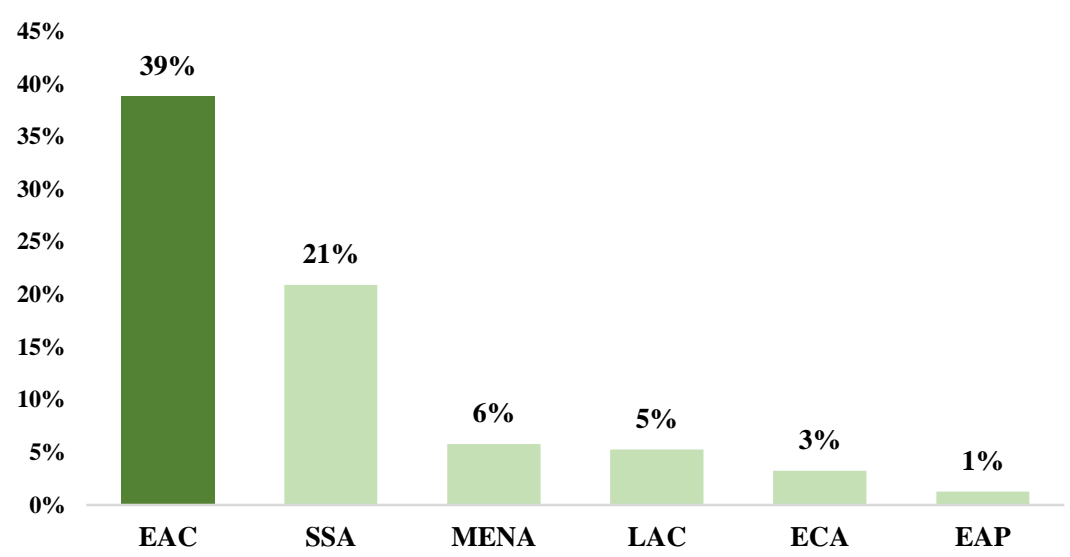

2. Usage of mobile money in East African Community

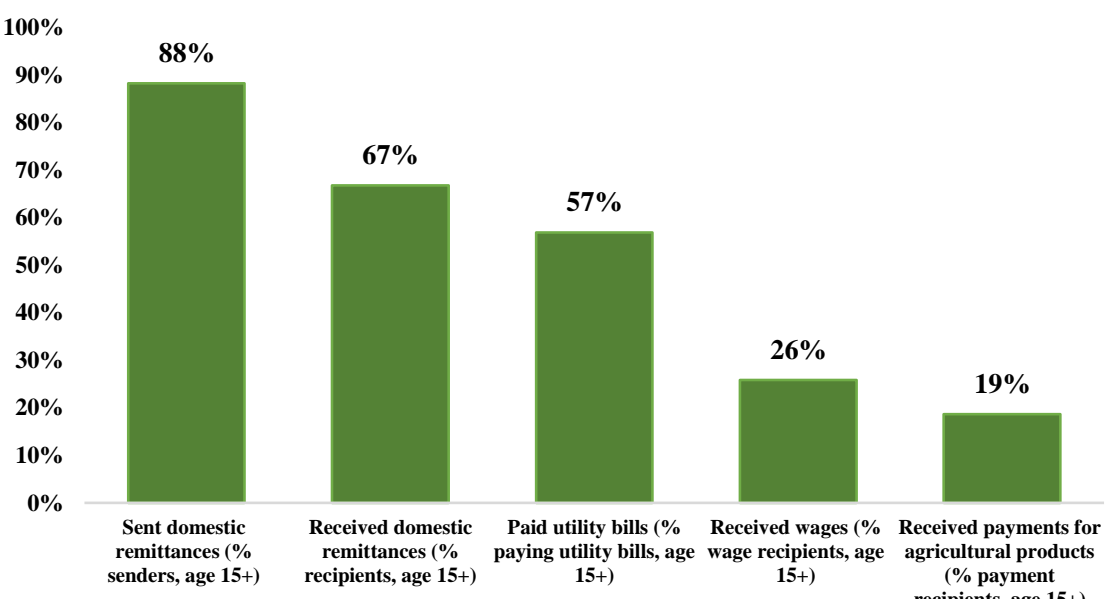

3. Mobile money account ( $\%$ age $15+)$ vs. Mobile banking (\% age $15+)$ and Financial account (\% age $15+)$ (Mobile banking: Used a mobile phone or the internet to access a financial institution account in the past year)

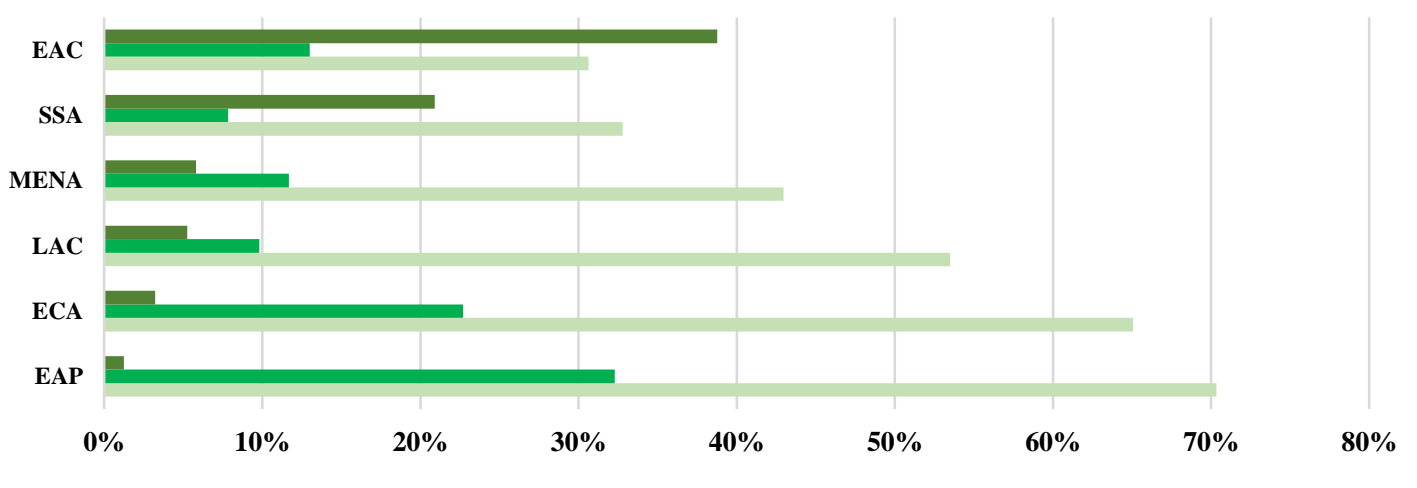

Mobile money account (\% age 15+) $\backsim$ Mobile banking (\% age 15+) $\backsim$ Financial institution account (\% age 15+)

Source: Authors' analysis using Global Financial Index Database and IMF Financial Access Survey.

Note: EAC=East African Community; SSA=Sub-Saharan Africa; MENA=Middle East and North Africa; LAC=Latin America and Caribbean; ECA=Europe and Central Asia; EAP=East Asia and Pacific. 